\title{
Strong Connections on Quantum Principal Bundles
}

\author{
Piotr M. HAJAC * \\ Mathematics Section, International Centre for Theoretical Physics, \\ Strada Costiera 11, 34014 Trieste, Italy.
}

6 May 1996

\begin{abstract}
A gauge invariant notion of a strong connection is presented and characterized. It is then used to justify the way in which a global curvature form is defined. Strong connections are interpreted as those that are induced from the base space of a quantum bundle. Examples of both strong and non-strong connections are provided. In particular, such connections are constructed on a quantum deformation of the Hopf fibration $S^{2} \rightarrow R P^{2}$. A certain class of strong $U_{q}(2)$-connections on a trivial quantum principal bundle is shown to be equivalent to the class of connections on a free module that are compatible with the $q$ dependent hermitian metric. A particular form of the Yang-Mills action on a trivial $U_{q}(2)-$ bundle is investigated. It is proved to coincide with the Yang-Mills action constructed by A. Connes and M. Rieffel. Furthermore, it is shown that the moduli space of critical points of this action functional is independent of $q$.
\end{abstract}

\section{Introduction}

Two of the mainstreams of Noncommutative Geometry concentrate around the notions of a projective module [12, 14] and of a quantum group [24, 38]. Quite recently (see [9, 22, 28]), the concept of a quantum principal bundle was systematically developed with quantum groups (Hopf algebras) in the role of structure groups. Hence, since both projective modules and quantum principal bundles serve as starting points for quantum geometric considerations, the conceptual framework provided by the notion of a quantum principal bundle has a good chance of unifying those two branches of Noncommutative Geometry.

In the classical differential geometry, it is hard to overestimate the interplay between Lie groups and $K$-theory. Therefore, it is natural to expect that establishing a similar interaction in the noncommutative case is necessary for better understanding of quantum geometry. It is already known that the classification of quantum principal bundles over manifolds depends only on the classical subgroups of quantum structure groups [19]. This leads to the following questions: Is the classification of (general) quantum principal bundles over noncommutative spaces richer then the classification of classical-group bundles over noncommutative spaces? Is there a bimodule that can be obtained as the bimodule of intertwiners (noncommutative

*On leave from: Department of Mathematical Methods in Physics, Warsaw University, ul. Hoża 74, Warsaw, 00-682 Poland. http://info.fuw.edu.pl/KMMF/ludzie_ang.html (E-mail: pmh@fuw.edu.pl) 
analogue of equivariant vector valued functions on a total space) only from a bundle with a noncommutative structure Hopf algebra? More generally, when does a deformation of a group into a quantum group entail essential consequences in the geometry (e.g., in the classification of bundles or in the Yang-Mills theory)?

Since this article is to a great extent a follow-up of [9], much of the mathematical and physical motivations listed there can also be considered as motivations for this work, and so will not be repeated here. Let us just emphasize that our main purpose is to specify and analyze a class of connections on quantum principal bundles (called strong connections) that enjoy some additional properties making them more like their classical counterparts, and (taking advantage of the notion of a strong connection) to discuss a link between the two approaches to noncommutative differential geometry based on quantum principal bundles and projective modules. The study of the precise relationship between those two approaches is thought of as a move towards answering the questions mentioned above.

We begin this article by fixing the notation and recalling the fundamentals of quantum bundles and Yang-Mills theory on projective modules. In the first section, in addition to this vocabulary review, we also study the definition of a quantum principal bundle using snake diagrams (see Remark 1.2 and Proposition 1.5). Taking advantage of Remark 1.2, we prove (Corollary 1.3) that the fundamental vector field compatibility condition (see Point 3 in Definition 1.1) implies its stronger version. (The latter version of the fundamental vector field compatibility condition was assumed in Example 4.11[9].)

The formalism used in this paper is a generalization of the corresponding formalism used in classical differential geometry. The calculations showing this, though often very instructive, are straightforward and we will not fully elaborate on that fact later on. Differential geometry on quantum principal bundles is still in the process of being born — the umbilical cord has hardly been cut yet - and it seems premature at this point to make precise categorical statements establishing the relationship between classical and quantum differential geometries. As Yu.I.Manin mentioned in a similar context (see p.86 in [27]), "Here, one should not act too hastily since even in supergeometry this program was started only recently and revealed both rich content and some puzzling new phenomena."

In Section 2, we define and provide examples of strong connections. Proposition 2.2 allows one to interpret strong connections on trivial quantum bundles as those induced from the base space, and to produce examples of strong connections in the case of trivial quantum bundles with the universal calculus. In the Introduction to the preliminary version of [9], one can read regarding inducing connection forms from the base space that "...in the general noncommutative or quantum case there would appear to be slightly more possibilities..." than in the classical case. Examples of connections that are not strong (and thus realize the just mentioned "quantum possibility") are supplied as well. More precisely, we construct both strong and non-strong connections on a very simple (yet rich enough) example of a 'discrete bundle' and on a quantum version of the Hopf fibration $S^{2} \rightarrow R P^{2}$. (As a byproduct of our considerations we obtain a $q$-deformation of $R P^{2}$.) It might be worthwhile to note that the latter construction does not employ the trivial-bundle or the quantum-group-quotient techniques. We end this section with presenting an example of a non-strong connection in the set-up of a strict 
monoidal category dual to the category of sets with Cartesian product.

In the subsequent section, we describe the action of (global) gauge transformations on the space of connections on a bundle with the universal differential calculus, and show that this action preserves the strongness of a connection.

In Section 4, we use the notion of a strong connection to justify the definition of a global curvature form. (We need to assume that a connection is strong if we want to show that its curvature form has certain properties that classical curvature forms possess automatically; e.g., the usual relation to the square of the exterior covariant derivative.)

In Section 5, we present a link between gauge theory on a quantum principal bundle and Yang-Mills theory on a projective module. First we show how, in the case of a free module, to incorporate the Yang-Mills action constructed in [13, 30 into the quantum bundle picture. Then, to obtain a Hermitian metric compatible connection on a free module from a strong $U_{q}(2)$ connection on a trivial quantum principal bundle, we mimic the classical geometry formula which permits one to determine the values of a connection form on a Hopf algebra of smooth functions on a matrix Lie group by knowing its values on the matrix of generators. (Note that usually one thinks of a connection form as a map sending smooth vector fields to elements of a Lie algebra, but we can also view it as a map from the Hopf algebra of smooth functions on a Lie group into the space of smooth 1-forms.) It turns out that the connections compatible with a particular $q$-dependent Hermitian structure can be identified with the strong $U_{q}(2)$ connections that satisfy certain condition. We close this section by concluding that, in the setting under consideration, the moduli space of critical points of $U_{q}(2)$ and $U(2)-$ Yang-Mills theory coincide. Thus, at least in this case, the $q$-deformation of the structure group alone has no essential bearing on the Yang-Mills theory. This seems to bring us a step closer to answering the question posed at the end of the first paragraph: One should expect geometrically interesting effects of the noncommutativity of a Hopf algebra in Yang-Mills theory only for non-trivial bundles (comodule algebras that are not crossed product algebras) or non-strong connections.

Finally, in the Appendix, we examine the advantages of adding a twist to the definition of a quantum associated bundle formulated in [9] and point out the possibility of using the axiomatic definition of a frame bundle to try to define its noncommutative analogue (cf. Section 5.1 in [9]).

\section{Preliminaries}

The notation used throughout this article is quite standard and not much different from that of [9]. Nevertheless, to eschew any possible misunderstanding or confusion, we enclose a table of basic notations:

$[. . .]_{X}$ an equivalence class defined by $X$

$\delta_{m, n}$ equals 1 iff $m=n$, and 0 otherwise (Kronecker symbol)

$\mathfrak{g} \quad$ Lie algebra of a Lie group $G$ 
$k \quad$ field of characteristic zero (except for Proposition 1.13)

$\otimes \quad$ tensor product over $k$

$\tau \quad$ flip $(\tau(u \otimes v):=v \otimes u)$

$\Omega^{1} A$ first order universal differential calculus $\left(\Omega^{1} A:=\operatorname{Ker} m_{A}, d a:=1 \otimes a-a \otimes 1\right)$

$\Omega A$ differential envelope of $A$

$\Omega(A)$ differential algebra over $A$ (i.e. a quotient of $\Omega A$ by some differential ideal)

$m_{X}$ multiplication on $X$, or in $X$ (We will simply write $m$ for the multiplication in a Hopf algebra.)

$\Delta$ comultiplication: $\Delta(a)=a_{(1)} \otimes a_{(2)}$ (Sweedler sigma notation with suppressed summation sign, cf. Section 1.2 in [35])

$\Delta_{n}$ comultiplication applied $n$ times (Due to coassociativity we do not have to remember where $\Delta$ is put in consecutive tensor products; $\left.\Delta_{n} a=a_{(1)} \otimes \cdots \otimes a_{(n+1)}.\right)$

$\eta_{Y} \quad$ unit map of an algebra $Y$ (often suppressed)

$\varepsilon \quad$ counit (unless otherwise obvious from the context)

$S \quad$ antipode, i.e. $a_{(1)} S\left(a_{(2)}\right)=\varepsilon(a)=S\left(a_{(1)}\right) a_{(2)}$

$A^{\text {op }}$ algebra identical with algebra $A$ as a vector space but with the multiplication defined by $m_{A^{o p}}=m_{A} \circ \tau$

$\rho_{R} \quad$ right coaction: $\rho_{R}(x)=x_{(0)} \otimes x_{(1)}$ (Sweedler sigma notation for comodules with suppressed summation sign, cf. p.32-3 in [35])

$\Delta_{R}$ right coaction on the 'total space' of a quantum principal bundle

$\Delta_{\mathcal{R}}$ right coaction on a right-covariant differential algebra of the 'total space' given by $\forall m \in \mathbb{N}: \Delta_{\mathcal{R}}\left(p_{0} d p_{1} \cdots\right.$ $\left(p_{0}\right)_{(0)} d\left(p_{1}\right)_{(0)} \cdots d\left(p_{m}\right)_{(0)} \otimes\left(p_{0}\right)_{(1)} \cdots\left(p_{m}\right)_{(1)}$, where for any $n \in\{0, \cdots, m\},\left(p_{n}\right)_{(0)} \otimes\left(p_{n}\right)_{(1)}=\Delta_{R} p_{n}$ (A differential algebra is right-covariant iff $\Delta_{\mathcal{R}}$ determined by the above formula is welldefined; cf. (21) and Section 4.2 in [9].)

$a d_{R}:=(i d \otimes m) \circ(i d \otimes S \otimes i d) \circ(\tau \otimes i d) \circ \Delta_{2}\left(\right.$ right adjoint coaction; $\left.a d_{R} a=a_{(2)} \otimes S\left(a_{(1)}\right) a_{(3)}\right)$

$P^{c o A}$ the space of right coinvariants $\left(P^{c o A}:=\left\{p \in P \mid \Delta_{R} p=p \otimes 1\right\}\right)$

$*_{\rho} \quad$ convolution: $\forall f \in \operatorname{Hom}_{k}(Q, X), g \in \operatorname{Hom}_{k}(A, Y): f *_{\rho} g=\mathrm{m} \circ(f \otimes g) \circ \rho_{R}$, where $\left(Q, \rho_{R}\right)$ is a right $A$-comodule and $\mathrm{m}: X \otimes Y \rightarrow Z$ is a multiplication map (If $\rho_{R}$ equals $\Delta_{\mathcal{R}}, \Delta_{R}$ or $\Delta$, we will use $*_{\mathcal{R}}, *_{R}$ or $*$ respectively to denote the corresponding convolution.)

$f^{-1}$ unless otherwise obvious from the context, convolution inverse of $f$, i.e.

$f^{-1}\left(a_{(1)}\right) f\left(a_{(2)}\right)=\left(f^{-1} * f\right)(a)=\varepsilon(a)=\left(f * f^{-1}\right)(a)=f\left(a_{(1)}\right) f^{-1}\left(a_{(2)}\right)$

(In general, one has: $x_{(0)} \otimes \cdots \otimes x_{(n)} \otimes f\left(x_{(n+1)}\right) g\left(x_{(n+2)}\right) \otimes x_{(n+3)} \otimes \cdots \otimes x_{(m)}$

$=x_{(0)} \otimes \cdots \otimes x_{(n)} \otimes(f * g)\left(x_{(n+1)}\right) \otimes x_{(n+2)} \otimes \cdots \otimes x_{(m-1)}$ and

$\left.\mathcal{F}\left(x_{(0)}\right) g\left(x_{(1)}\right) \otimes x_{(2)} \otimes \cdots \otimes x_{(m)}=\left(\mathcal{F} *_{\rho} g\right)\left(x_{(0)}\right) \otimes x_{(1)} \otimes \cdots \otimes x_{(m-1)}.\right)$ 
All algebras are assumed to be unital and associative. Now, let us recall the basic notions and constructions of [9] necessary to establish the language used in this paper.

Definition 1.1 (4.9 [9]) Let $P$ be an algebra over a field $k, A$ a Hopf algebra over the same field, $N_{P} \subseteq \Omega^{1} P$ a $P$-bimodule defining the first order differential calculus $\Omega^{1}(P), M_{A} \subseteq \operatorname{Ker} \varepsilon$ an ad $_{R^{-}}$invariant right ideal defining the bicovariant differential calculus $\Omega^{1}(A)$, and

$$
\Delta_{R}: P \longrightarrow P \otimes A
$$

an algebra homomorphism making $P$ a right $A$-comodule algebra.

Then $\left(P, A, \Delta_{R}, N_{P}, M_{A}\right)$ is called a quantum principal bundle iff:

1. $\mathrm{T}_{R}: P \otimes P \ni t \stackrel{\text { def. }}{\longmapsto}(m \otimes i d) \circ\left(i d \otimes \Delta_{R}\right) t \in P \otimes A$ is a surjection (freeness condition),

2. $\Delta_{\mathcal{R}}\left(N_{P}\right) \subseteq N_{P} \otimes A$ (right covariance of the differential structure),

3. $\mathrm{T}_{R}\left(N_{P}\right) \subseteq P \otimes M_{A}$ (fundamental vector field compatibility condition),

4. Ker $\top \subseteq P \Omega^{1}\left(P^{c o A}\right) P($ exactness condition $)$, where $\Omega^{1}\left(P^{c o A}\right):=\Omega^{1} P^{c o A} /\left(N_{P} \cap \Omega^{1} P^{c o A}\right)$

$$
\text { and } \quad \mathrm{T}: \Omega^{1}(P) \ni[\alpha]_{N_{P}} \stackrel{\text { def. }}{\longmapsto}\left(\left(i d \otimes \pi_{A}\right) \circ \mathrm{T}_{R}\right) \alpha \in P \otimes\left(\operatorname{Ker} \varepsilon / M_{A}\right)
$$

(the $\operatorname{map} \pi_{A}: \operatorname{Ker} \varepsilon \rightarrow \operatorname{Ker} \varepsilon / M_{A}$ is the canonical projection, and $\alpha \in \operatorname{Ker} m_{P}$ ).

For simplicity, as well as to emphasize the analogy with the classical situation, a quantum principal bundle is often denoted by $P(B, A)$, where $B:=P^{c o} A$ is the 'base space' of the bundle. The map $\mathrm{T}$ (denoted by $\widetilde{\sim}_{N_{P}}$ in 9 ) can be more explicitly described by the formula

$$
\mathrm{T}(p d q)=p q_{(0)} \otimes\left[q_{(1)}\right]_{M_{A}}-p q \otimes 1
$$

Remark 1.2 Let $\mathrm{T}_{U}:$ Ker $m_{P} \rightarrow P \otimes$ Ker $\varepsilon$ and $\mathrm{T}_{N M}: N_{P} \rightarrow P \otimes M_{A}$ be the appropriate restrictions of $\mathrm{T}_{R}$. It is straightforward to check that the following diagrams are commutative diagrams (of left $P$-modules) with exact rows and columns:

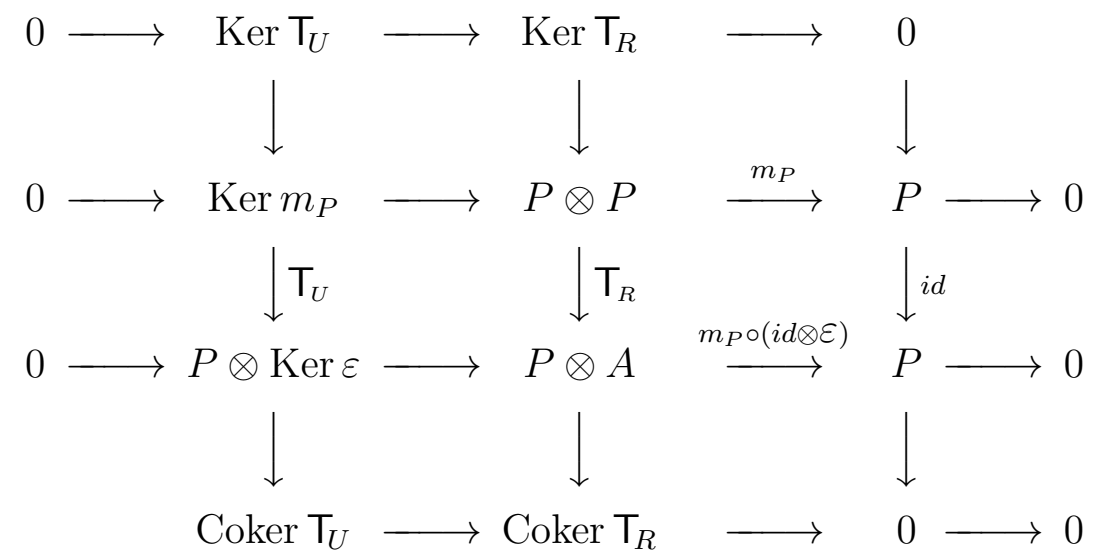




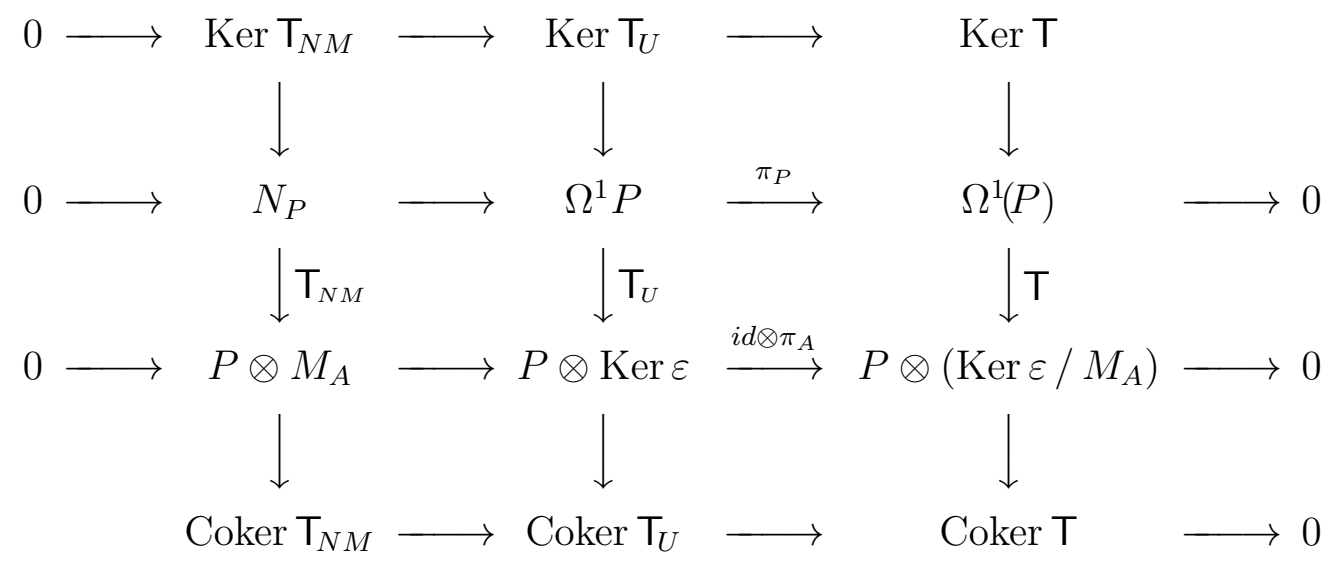

Applying the Snake Lemma (e.g., see Section 1.2 in [5]) to both diagrams, we obtain the following two exact sequences:

$$
\begin{aligned}
& 0 \longrightarrow \mathrm{Ker}_{U} \longrightarrow \operatorname{Ker} \mathrm{T}_{R} \longrightarrow 0 \longrightarrow \text { Coker } \mathrm{T}_{U} \longrightarrow \text { Coker } \mathrm{T}_{R} \longrightarrow 0 \\
& 0 \longrightarrow \operatorname{Ker} \mathrm{T}_{N M} \longrightarrow \operatorname{Ker}_{U} \longrightarrow \operatorname{Ker} \mathrm{T} \longrightarrow \text { Coker }_{N M} \longrightarrow \text { Coker }_{U} \longrightarrow \text { Coker } \mathrm{T} \longrightarrow 0
\end{aligned}
$$

Observe that the freeness condition means exactly that Coker $\mathrm{T}_{R}=0$, which, by (3), is equivalent to Coker $\mathrm{T}_{U}=0$ (see (33) in [9]). Note also that $\operatorname{Ker} \mathrm{T}_{N M}=N_{P} \cap \operatorname{Ker} \mathrm{T}_{U}$.

Corollary 1.3 Let $\left(P, A, \Delta_{R}, N_{P}, M_{A}\right)$ be a quantum principal bundle. Then $\mathrm{T}_{R}\left(N_{P}\right)=P \otimes M_{A}$ (cf. Example 4.11 in [9] and the discussion below it).

Proof. From the exactness condition, we know that $\operatorname{Ker} \mathrm{T}=\pi_{P}\left(P \Omega^{1} B . P\right)$. On the other hand, since $P \Omega^{1} B . P \subseteq \operatorname{Ker} \mathrm{T}_{U}$ and the map $\pi_{U}: \operatorname{Ker}_{U} \rightarrow \operatorname{Ker} \mathrm{T}$ in (雨) is a restriction of $\pi_{P}$ to $\operatorname{Ker} \mathrm{T}_{U}$, we can conclude that $\pi_{U}$ is surjective. Consequently, by the freeness condition and the exactness of (四), Coker $\mathrm{T}_{N M}=0$, i.e. $\mathrm{T}_{R}\left(N_{P}\right)=P \otimes M_{A}$.

The above corollary makes the following definition of a trivial quantum principal bundle equivalent to the definition proposed in Example 4.11 in [9].

Definition 1.4 A quantum principal bundle $\left(P, A, \Delta_{R}, N_{P}, M_{A}\right)$ is called trivial iff there exists a convolution invertible map (trivialization) $\Phi \in \operatorname{Hom}_{k}(A, P)$ such that

$$
\Delta_{R} \circ \Phi=(\Phi \otimes i d) \circ \Delta
$$

(i.e. $\Phi$ is right-covariant) and $\Phi(1)=1$. In such a case, $P$ is also called a crossed product or cleft extension (see p.273 in 39 ). 
Definition 1.5 (1.1 32]) Let $P$ be a right $A$-comodule algebra and $B$ be the algebra of all right coinvariants. The comodule $P$ is called an A-Galois extension iff the canonical left P-algebra and right $A$-coalgebra map

$$
\mathrm{T}_{B}:=\left(m_{P} \otimes i d\right) \circ\left(i d \otimes_{B} \Delta_{R}\right): P \otimes_{B} P \ni p \otimes_{B} q \longmapsto p q_{(0)} \otimes q_{(1)} \in P \otimes A
$$

is bijective.

Proposition 1.6 円et $P, A$ and $B$ be as above. A comodule algebra $P$ is an A-Galois extension if and only if $P(B, A)$ is a quantum principal bundle with the universal calculus.

Proof. Consider the following commutative diagram (of left $P$-modules) with exact rows and columns:

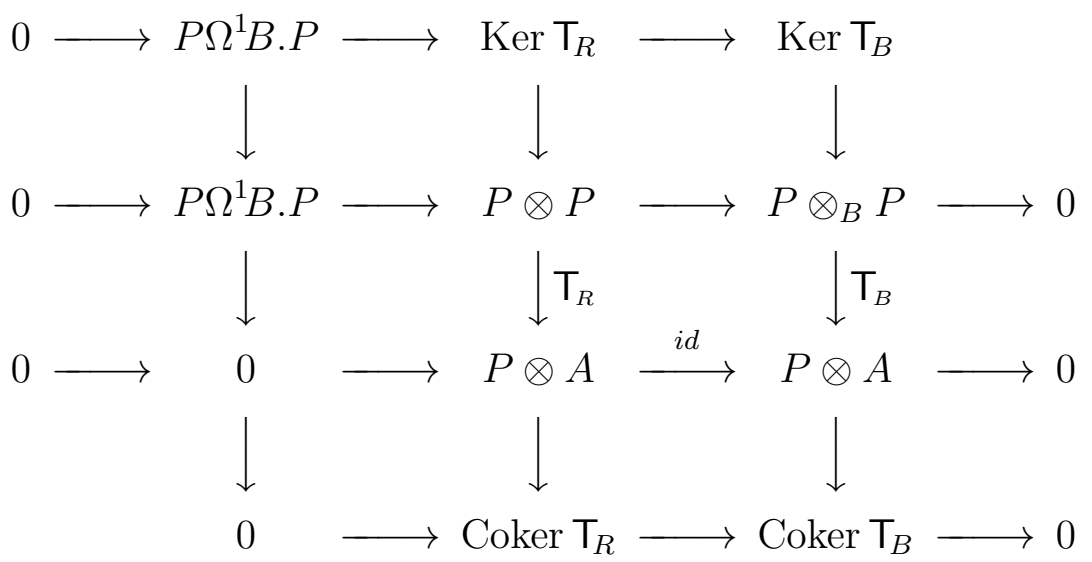

Again, we can apply the Snake Lemma to obtain an exact sequence

$$
0 \longrightarrow P \Omega^{1} B . P \longrightarrow \operatorname{Ker}_{R} \longrightarrow \operatorname{Ker}_{B} \longrightarrow 0 \longrightarrow \text { Coker }_{R} \longrightarrow \text { Coker }_{B} \longrightarrow 0 \text {. }
$$

Assume first that $P$ is an $A$-Galois extension. Then $\operatorname{Ker} \mathrm{T}_{B}=0=\operatorname{Coker} \mathrm{T}_{B}$, and, from the exactness of (7), we can infer that Coker $\mathrm{T}_{R}=0$ (freeness condition) and $\operatorname{Ker} \mathrm{T}_{R}=P \Omega^{1} B . P$. On the other hand, by the exactness of (3), we have $\operatorname{Ker} \mathrm{T}_{U}=\operatorname{Ker} \mathrm{T}_{R}$. Hence the exactness condition follows, and we can conclude that $P(B, A)$ is a quantum principal bundle with the universal calculus.

Conversely, assume that $P(B, A)$ is a quantum principal bundle with the universal calculus. Then Ker $\mathrm{T}_{R}=\operatorname{Ker} \mathrm{T}_{U}=P \Omega^{1} B . P$ and Coker $\mathrm{T}_{R}=0$. Consequently, again due to the exactness of (7), we have that $\operatorname{Ker} \mathrm{T}_{B}=0=$ Coker $\mathrm{T}_{B}$, i.e. $P$ is an $A$-Galois extension.

Definition $1.7([9])$ A left $P$-module projection $\Pi$ on $\Omega^{1}(P)$ is called a connection on $P(B, A)$ iff

1 This proposition is implicitly proved in [7] (see Lemma 3.2 and the text above it). The diagrammatic proof presented here was created during the author's discussion with Markus Pflaum. 
1. $\operatorname{Ker} \Pi=\Omega_{\text {hor }}^{1}(P)$ ( $\operatorname{Im} \Pi$ is called the space of vertical forms),

2. $\Delta_{\mathcal{R}} \circ \Pi=(\Pi \otimes i d) \circ \Delta_{\mathcal{R}}($ right covariance $)$.

Due to Proposition 4.10 in [9], a connection form can be defined in the following way:

Definition 1.8 A $k$-homomorphism $\omega: A \rightarrow \Omega^{1}(P)$ is called a connection form on $P(B, A)$ iff it satisfies the following properties:

1. $\omega\left(k \oplus M_{A}\right)=0$ (compatibility with the differential structure),

2. $\mathrm{T} \circ \omega=\left(i d \otimes \pi_{A}\right) \circ(1 \otimes(i d-\varepsilon))$ (fundamental vector field condition),

3. $\Delta_{\mathcal{R}} \circ \omega=(\omega \otimes i d) \circ \operatorname{ad}_{R}($ right adjoint covariance $)$.

For every $P(B, A)$, there is a one-to-one correspondence between connections and connection forms. In particular, the connection $\Pi^{\omega}$ associated to a connection form $\omega$ is given by the formula: $\Pi^{\omega}=m_{\Omega^{1}(P)} \circ(i d \otimes \omega) \circ \mathrm{T}((47)$ in [9] $)$. Since $\Pi^{\omega}$ is a left $P$-module homomorphism, to calculate $\Pi^{\omega}$ it suffices to know its values on exact forms, and on exact forms (47) [9 simplifies to

$$
\Pi^{\omega} \circ d=i d *_{R} \omega
$$

The following four definitions are based on Appendix A[9].

Definition 1.9 Let $\Omega(P)$ be any differential algebra having $\Omega^{1}(P)$ as in Definition 1.1. For all $n \in \mathbb{N}$, the space $\left(P \Omega^{1}(B) P\right)^{n}$ is called the space of horizontal $n$-forms and is denoted by $\Omega_{\text {hor }}^{n}(P)$. The space of horizontal 0 -forms is identified with $P$.

Definition 1.10 Let $\Omega(B)$ be the differential algebra obtained by the restriction of $\Omega(P)$. For all $n \in \mathbb{N}$, the space $\Omega^{n}(B) P$ is called the space of strongly horizontal $n$-forms and is denoted by $\Omega_{\text {shor }}^{n}(P)$. The space of strongly horizontal 0-forms is identified with $P$.

Note that in the classical case $\Omega_{h o r}^{*}(P)$ and $\Omega_{\text {shor }}^{*}(P)$ coincide.

Definition 1.11 Let $\left(V, \rho_{R}\right)$ be a right $A^{\text {op }}$-comodule algebra (see Remark A.2). Then $\varphi \in \operatorname{Hom}_{k}(V, \Omega(P))$ is called a pseudotensorial form on $P$ iff

$$
\Delta_{\mathcal{R}} \circ \varphi=(\varphi \otimes i d) \circ \rho_{R}
$$

A pseudotensorial form taking values in $\Omega_{\text {hor }}^{*}(P)$ (in $\Omega_{\text {shor }}^{*}(P)$ ) is called a tensorial (strongly tensorial) form on $P$. The space of all pseudotensorial, tensorial and strongly tensorial $n$-forms $(n \geq 0)$ will be denoted by $P T_{\rho}\left(V, \Omega^{n}(P)\right), T_{\rho}\left(V, \Omega^{n}(P)\right)$ and $S T_{\rho}\left(V, \Omega^{n}(P)\right)$ respectively. 
Definition 1.12 ((68)[9]) Let $\Pi$ be a connection on $P$. The $k$-homomorphism $D$ from $\Omega^{*} P$ to $\Omega_{\text {hor }}^{*+1} P$ given by

$$
D: p_{0} d p_{1} \cdots d p_{n} \longmapsto(i d-\Pi)\left(d p_{0}\right) \cdots(i d-\Pi)\left(d p_{n}\right)
$$

where $n \geq 0$, is called the exterior covariant derivative associated to $\Pi$.

To complete this vocabulary review, we recall some basic definitions used in the Yang-Mills theory on projective modules. We choose here right rather than left modules, but one should bear in mind that the formulation of this formalism for left modules is analogous.

Proposition 1.13 (cf. p.369 in [34]) Let $\mathcal{B}$ be an associative unital algebra over a commutative ring $k$. Let $\mathcal{L}_{\|}$be a $k$-Lie subalgebra of the space of all $k$-derivations of $\mathcal{B}$, and let $\mathcal{E}$ be any right $\mathcal{B}$-module admitting a connection. If $\Omega(\mathcal{B})$ is a differential graded subalgebra of $\mathcal{B} \oplus \bigoplus_{\backslash \geq \infty}^{\infty} \operatorname{Hom}_{\|}\left(\wedge^{\backslash} \mathcal{L}_{\|}, \mathcal{B}\right)$ with the differential given by (see the first section in [18]):

$$
\begin{aligned}
(d \alpha)\left(X_{0}, X_{1}, \cdots, X_{n}\right) & =\sum_{0 \leq i \leq n}(-)^{i} X_{i} \alpha\left(X_{0}, \cdots, X_{i-1}, X_{i+1}, \cdots, X_{n}\right) \\
& +\sum_{0 \leq r<s \leq n}(-)^{r+s} \alpha\left(\left[X_{r}, X_{s}\right], X_{0}, \cdots, X_{r-1}, X_{r+1}, \cdots, X_{s-1}, X_{s+1}, \cdots, X_{n}\right),
\end{aligned}
$$

then

$$
\forall \xi \in \mathcal{E}, \mathcal{X}, \mathcal{Y} \in \mathcal{L}_{\|}:\left(\nabla^{\in} \xi\right)(\mathcal{X}, \mathcal{Y})=\left(\left[\nabla_{\mathcal{X}}, \nabla_{\mathcal{Y}}\right]-\nabla_{[\mathcal{X}, \mathcal{Y}]}\right)(\xi)
$$

where, as in the classical differential geometry, $\nabla_{Z} \xi$ denotes $(\nabla \xi)(Z)$.

Proof. Straightforward.

Definition 1.14 ([13, 30]) Let $\mathcal{L}$ be a finite dimensional Lie subalgebra of Der $B$, let $\left\{X_{l}\right\}_{l \in\{1, \ldots, \operatorname{dim} \mathcal{L}\}}$ be a basis of $\mathcal{L}$, and let $\Omega(B)$ be a differential algebra defined as in Proposition 1.13. A bilinear form

$$
\{,\}:\left(\operatorname{End}_{B}(\mathcal{E}) \otimes_{\mathcal{B}} \Omega^{\in}(\mathcal{B})\right) \times\left(\operatorname{End}_{\mathcal{B}}(\mathcal{E}) \otimes_{\mathcal{B}} \Omega^{\in}(\mathcal{B})\right) \longrightarrow \operatorname{End}_{\mathcal{B}}(\mathcal{E})
$$

is defined by

$$
\left\{\psi_{1}, \psi_{2}\right\}=\sum_{i<j} \psi_{1}\left(X_{i} \wedge X_{j}\right) \psi_{2}\left(X_{i} \wedge X_{j}\right)
$$

Definition 1.15 (cf. p.553-4 in [12]) Let $\mathcal{E}$ be a finitely generated projective right B-module.

1. A linear map $\nabla: \mathcal{E} \otimes_{\mathcal{B}} \otimes^{*}(\mathcal{B}) \rightarrow \mathcal{E} \otimes_{\mathcal{B}} \otimes^{*+\infty}(\mathcal{B})$ is called a connection on $\mathcal{E}$ iff

$$
\forall \xi \in \mathcal{E}, \alpha \in \otimes(\mathcal{B}): \nabla\left(\xi \otimes_{\mathcal{B}} \alpha\right)=(\nabla \xi) \alpha+\xi \otimes_{\mathcal{B}}\lceil\alpha
$$


2. The endomorphism $\nabla^{2} \in \operatorname{End}_{\Omega(B)}\left(\mathcal{E} \otimes_{\mathcal{B}} \Omega(\mathcal{B})\right)$ is called the curvature of a connection $\nabla$.

3. If $B$ is a *-algebra and $\mathcal{E}$ is equipped with a Hermitian metric $\langle\rangle:, \mathcal{E} \times \mathcal{E} \rightarrow \mathcal{B}$, then we say that a connection on $\mathcal{E}$ is compatible with this Hermitian metric iff

$$
\forall \xi, \eta \in \mathcal{E}:\lceil\langle\xi, \eta\rangle=\langle\nabla \xi, \eta\rangle+\langle\xi, \nabla \eta\rangle
$$

Remark 1.16 The group $U(\mathcal{E}):=\left\{\mathcal{U} \in \operatorname{End}_{\mathcal{B}}(\mathcal{E}) \mid \forall \xi, \eta \in \mathcal{E}:\langle\mathcal{U} \xi, \mathcal{U} \eta\rangle=\langle\xi, \eta\rangle\right\}$ of unitary automorphisms of $\mathcal{E}$ acts on the space of connections in the following way: $\nabla \mapsto U \nabla U^{*}$. This action maps compatible connections to compatible connections (see near the end of Section 1 in [13]).

Remark 1.17 The sign in the formula (11) depends on whether we want $d\left(a^{*}\right)=(d a)^{*}$ or $d\left(a^{*}\right)=-(d a)^{*}$. We have ' + ' in (11) because here we choose that $d$ commute with *.

Definition $1.18\left([\overline{13}\right.$, 30] $)$ A trace $\mathcal{T}_{\mathcal{E}}: \operatorname{End}_{\mathcal{B}}(\mathcal{E}) \longrightarrow \|$ is given by $\mathcal{T}_{\mathcal{E}}(\xi\langle\zeta,\rangle)=.\mathcal{T}_{\mathcal{B}}\langle\zeta, \xi\rangle$, where $\mathcal{T}_{\mathcal{B}}$ is a trace on $B$.

Corollary 1.19 Let $\mathcal{E}=\mathcal{B} \backslash$ for some $n \in \mathbb{N}$, and $N \in \operatorname{End}_{B}\left(B^{n}\right)=M_{n}(B)$. Then

$$
\mathcal{T}_{\mathcal{E}}(\mathcal{N})=\left(\mathcal{T}_{\mathcal{B}} \circ \mathcal{T} \nabla\right)(\mathcal{N})
$$

where $\operatorname{Tr}$ is the usual matrix trace.

Proof. Straightforward.

Definition 1.20 ([13, [30], cf. [12, 17, 18]) Let $\nabla$ be a connection on $\mathcal{E}$. The function $Y M$ given by the formula

$$
Y M(\nabla)=-\mathcal{T}_{\mathcal{E}}\left\{\times_{\nabla}, \times_{\nabla}\right\}
$$

where $\Theta$ is defined by $\Theta_{\nabla}(X, Y)=\left[\nabla_{X}, \nabla_{Y}\right]-\nabla_{[X, Y]}$, is called the Yang-Mills action functional.

Example 1.21 Let $G$ be a compact Lie group and $B=C^{\infty}(G)$. Let $W$ be a finite dimensional vector space, $X(G, G L(W))$ a principal bundle, $\mathcal{E}=\Gamma\left(\mathcal{X}(\mathcal{G}, \mathcal{G} \mathcal{L}(\mathcal{W})) \times_{\rangle} \mathcal{W}\right)$ the projective module of the smooth sections of the $i d$-associated vector bundle, and $g$ a Riemannian metric on $G$. (The choice of a metric on $\mathcal{E}$ makes no difference.) Then, for any connection form $\omega$ on $X(G, G L(W))$, one has $Y M\left(\nabla^{\omega}\right)=-\int_{G} \operatorname{Tr}\left(F^{\omega} \wedge \star F^{\omega}\right)$, where $\nabla^{\omega}$ is the covariant derivative (connection on $\mathcal{E}$ ) associated to $\omega$, the symbol $\star$ denotes the Hodge star associated to $g$, and $F^{\omega}$ is the curvature 2 -form of $\omega$ (see pages 336,337 and 360 in «4). 


\section{Strong Connections}

We can now proceed to formulate the notion of a strong connection. This notion will be used to justify the definition of a global curvature form. It will also be needed for characterizing these connections on a trivial $U_{q}(2)$-bundle that correspond to the hermitian connections on the free module associated with this bundle.

Definition 2.1 Let $\left(P, A, \Delta_{R}, N_{P}, M_{A}\right)$ be a quantum principal bundle. A connection $\Pi$ on $P$ is called strong iff $(i d-\Pi)(d P) \subseteq \Omega_{\text {shor }}^{1}(P)$ (see also Remark 4.3).

For every connection $\Pi$, the left $P$-module homomorphism $(i d-\Pi)$ maps exact 1 -forms to horizontal, but not necessarily to strongly horizontal, 1-forms. A strong connection $\Pi$ is defined by requiring that $(i d-\Pi)$ sends exact 1 -forms to strongly horizontal 1 -forms. It turns out that, for the trivial quantum principal bundles, the strongness of a connection means that the connection is induced from the base space of a bundle. (A similar fact is described in Lemma 6.11 in [20].) More precisely, let $\beta \in \operatorname{Hom}_{k}\left(A, \Omega^{1}(B)\right)$ be the map given by the formula

$$
\beta=\Phi * \omega * \Phi^{-1}+\Phi *\left(d \circ \Phi^{-1}\right)
$$

(This formula can be obtained by solving formula (37)[9] for $\beta$ and extending the solution to a general differential calculus.) It is straightforward to check that in the classical case the thus defined $\beta$ corresponds exactly to the pullback of a connection 1-form with respect to the section associated with a given trivialization. Therefore, we can think of $\beta$ as a noncommutative analog of the aforementioned pullback of a connection form (see also Remark 2.6). (For a discussion of connection forms which can be understood as elements of $\operatorname{Hom}_{k}\left(A / k, \Omega^{1}(B)\right)$, and which are also called quantum group gauge fields, see Section 3 in [9].) With the help of (13), we can characterize the class of strong connections on any trivial quantum principal bundle in the following way:

Proposition 2.2 Let $\left(P, A, \Delta_{R}, N_{P}, M_{A}\right)$ be a trivial quantum principal bundle with a trivialization $\Phi$ and a connection form $\omega$, and let $\beta$ be as above. Then $\Pi^{\omega}$ is strong if and only if $\beta(A) \subseteq \Omega^{1}(B)$.

Proof. It is known (e.g., see (27) in [9]) that

$$
\forall p \in P \exists \sum_{i} b_{i} \otimes a_{i} \in B \otimes A: p=\sum_{i} b_{i} \Phi\left(a_{i}\right) \text {. }
$$

Using this fact, formulas (8), (5), (37) [9], and the Leibniz rule it can be calculated that

$$
\left(i d-\Pi^{\omega}\right)(d p)=\sum_{i}\left(d b_{i} . \Phi\left(a_{i}\right)-b_{i}(\beta * \Phi)\left(a_{i}\right)\right) .
$$

(This calculation appeared in the preliminary version of [9].) Clearly, $\beta(A) \subseteq \Omega^{1}(B)$ implies that $\Pi^{\omega}$ is a strong connection. To prove the reverse implication, we will use the following lemmas and corollary: 
Lemma 2.3 Let $\left\{\left(Q_{i}, \rho_{i}\right)\right\}_{i \in I}$ and $\left(\mathcal{C}, \rho_{\mathcal{C}}\right)$ be right $A$-comodules, and $\left\{V_{i j}\right\}_{i, j \in I}$ be vector spaces, where $I$ is a non-empty finite set. Assume also that, for all $i, j \in I$, we have multiplication maps

$$
\begin{aligned}
& m_{i j}: Q_{i} \otimes Q_{j} \longrightarrow V_{i j} \\
& m_{i j, l}: V_{i j} \otimes Q_{l} \longrightarrow \mathcal{C} \\
& m_{i, j l}: Q_{i} \otimes V_{j l} \longrightarrow \mathcal{C}
\end{aligned}
$$

satisfying the associativity condition

$$
\forall i, j, l \in I: m_{i j, l} \circ\left(m_{i j} \otimes i d\right)=m_{i, j l} \circ\left(i d \otimes m_{j l}\right)
$$

and that coactions $\left\{\rho_{i}, \rho_{\mathcal{C}}\right\}_{i \in I}$ are compatible in the following sense:

$$
\forall i, j, l \in I, q_{i} \in Q_{i}, q_{j} \in Q_{j}, q_{l} \in Q_{l}: \rho_{\mathcal{C}}\left(q_{i} q_{j} q_{l}\right)=\rho_{i}\left(q_{i}\right) \rho_{j}\left(q_{j}\right) \rho_{l}\left(q_{l}\right)
$$

Then, for any $i, j, l \in I$, if $\kappa_{i} \in \operatorname{Hom}_{k}\left(A, Q_{i}\right), \varpi_{j} \in \operatorname{Hom}_{k}\left(A, Q_{j}\right)$ and $\lambda_{l} \in \operatorname{Hom}_{k}\left(A, Q_{l}\right)$ are homomorphisms with the right-covariance properties:

$$
\begin{aligned}
& \rho_{i} \circ \kappa_{i}=\left(\kappa_{i} \otimes i d\right) \circ \Delta, \\
& \rho_{j} \circ \varpi_{j}=\left(\varpi_{j} \otimes i d\right) \circ a d_{R}, \\
& \rho_{l} \circ \lambda_{l}=\left(\lambda_{l} \otimes S\right) \circ \tau \circ \Delta,
\end{aligned}
$$

the homomorphism $\kappa_{i} * \varpi_{j} * \lambda_{l}$ is right-invariant, i.e.

$$
\rho_{\mathcal{C}} \circ\left(\kappa_{i} * \varpi_{j} * \lambda_{l}\right)=\left(\kappa_{i} * \varpi_{j} * \lambda_{l}\right) \otimes 1
$$

Proof. A direct sigma notation computation proves this lemma.

Corollary 2.4 Let $\omega$ be a connection form on a trivial quantum principal bundle with a trivialization $\Phi$. The map $\beta$ given by (13) takes values in right-invariant differential forms, i.e.

$$
\Delta_{\mathcal{R}} \circ \beta=\beta \otimes 1
$$

Proof. Taking advantage of the formula

$$
\Delta_{R} \circ \Phi^{-1}=\left(\Phi^{-1} \otimes S\right) \circ \tau \circ \Delta
$$

one can deduce from Lemma 2.3 that

$$
\Delta_{\mathcal{R}} \circ\left(\Phi * \omega * \Phi^{-1}\right)=\left(\Phi * \omega * \Phi^{-1}\right) \otimes 1 .
$$

(Observe that, since

$$
\left(\left(\Phi^{-1} \otimes S\right) \circ \tau \circ \Delta\right) *\left(\Delta_{R} \circ \Phi\right)=\eta_{P \otimes A} \circ \varepsilon=\left(\Delta_{R} \circ \Phi\right) *\left(\left(\Phi^{-1} \otimes S\right) \circ \tau \circ \Delta\right)
$$


formula (28) 9] follows by the same argument as used in the proof of Proposition 3.2.) Furthermore, as

$$
\begin{aligned}
& \Delta_{\mathcal{R}} \circ\left(d \circ \Phi^{-1}\right) \\
& \left.=(d \otimes i d) \circ \Delta_{R} \circ \Phi^{-1} \quad \text { (see the beginning of Section } 1 \text { for this property of } \Delta_{\mathcal{R}}\right) \\
& =\left(\left(d \circ \Phi^{-1}\right) \otimes S\right) \circ \tau \circ \Delta,
\end{aligned}
$$

and $\eta_{P} \circ \varepsilon$ is $a d_{R^{-c o v a r i a n t}}$ - i.e. $\quad \Delta_{R} \circ \eta_{P} \circ \varepsilon=\left(\left(\eta_{P} \circ \varepsilon\right) \otimes i d\right) \circ a d_{R}$ - it also follows from Lemma 2.3 that

$$
\Delta_{\mathcal{R}} \circ\left(\Phi *\left(\eta_{P} \circ \varepsilon\right) *\left(d \circ \Phi^{-1}\right)\right)=\left(\Phi *\left(\eta_{P} \circ \varepsilon\right) *\left(d \circ \Phi^{-1}\right)\right) \otimes 1
$$

Combining formulas (15) and (16) we get the assertion of the corollary.

Lemma 2.5 Let $\left(Q, \rho_{0}\right)$ be a right $A$-comodule, and let $\left\{\left(Q_{i}, \rho_{i}\right)\right\}_{i \in\{1,2\}}, V_{12}$ and $m_{12}$ be as in Lemma 2.3. If $f_{1} \in \operatorname{Hom}_{k}\left(Q, Q_{1}\right)$ is right-covariant (i.e. $\left.\rho_{1} \circ f_{1}=\left(f_{1} \otimes i d\right) \circ \rho_{0}\right)$ and $f_{2} \in \operatorname{Hom}_{k}\left(A, Q_{2}\right)$, then

$$
\left(i d *_{\rho_{1}} f_{2}\right) \circ f_{1}=f_{1} *_{\rho_{0}} f_{2} .
$$

Proof. Straightforward.

Assume now that $\Pi^{\omega}$ is strong. Then, by setting $p=\Phi(a)$ in (14), we obtain:

$$
(\beta * \Phi)(A) \subseteq \Omega_{\text {shor }}^{1}(P)
$$

Furthermore, it is a general fact that

$$
\left(i d *_{\mathcal{R}} \Phi^{-1}\right)\left(\Omega_{\text {shor }}^{1}(P)\right) \subseteq \Omega^{1}(B)
$$

Indeed, for any $b \in B, p \in P$,

$$
\left(i d *_{\mathcal{R}} \Phi^{-1}\right)(d b . p)=\left(m_{\Omega^{1}(P)} \circ\left(i d \otimes \Phi^{-1}\right)\right)\left(d b_{(0)} p_{(0)} \otimes b_{(1)} p_{(1)}\right)=d b . \mathrm{S}_{\Phi}(p),
$$

where

$$
\mathrm{S}_{\Phi}: P \ni p \stackrel{\text { def. }}{\longmapsto}\left(i d *_{R} \Phi^{-1}\right)(p)=p_{(0)} \Phi^{-1}\left(p_{(1)}\right) \in B
$$

is a left $B$-module homomorphism that can be interpreted as the section of $P(B, A)$ associated with the trivialization $\Phi$ (see the subsequent remark, cf. Section 3.1 in [10]). Taking again advantage of the formula (28)[9] (see the proof of the Corollary 2.4) we can infer that

$$
\Delta_{R} \circ\left(i d *_{R} \Phi^{-1}\right)=\left(i d *_{R} \Phi^{-1}\right) \otimes 1
$$

(cf. the second calculation in the proof of Proposition A.7 in [9]). Hence $\mathrm{s}_{\Phi}$ indeed maps into $B$, and (18) follows as claimed. Combining (17) and (18) one can conclude that

$$
\left(\left(i d *_{\mathcal{R}} \Phi^{-1}\right) \circ(\beta * \Phi)\right)(A) \subseteq \Omega^{1}(B) .
$$


On the other hand, taking into account Corollary 2.4, one can see that $\beta * \Phi$ is right-covariant:

$$
\begin{aligned}
& \Delta_{\mathcal{R}} \circ(\beta * \Phi) \\
& =\left(\left(\Delta_{\mathcal{R}} \circ \beta\right) *\left(\Delta_{R} \circ \Phi\right)\right) \\
& =m_{\Omega^{1}(P) \otimes A} \circ((\beta \otimes 1) \otimes(\Phi \otimes i d)) \circ \Delta_{2} \\
& =((\beta * \Phi) \otimes i d) \circ \Delta .
\end{aligned}
$$

Hence, by Lemma 2.5,

$$
\left(i d *_{\mathcal{R}} \Phi^{-1}\right) \circ(\beta * \Phi)=(\beta * \Phi) * \Phi^{-1}=\beta
$$

and (21) reduces to $\beta(A) \subseteq \Omega^{1}(B)$, as needed.

Remark 2.6 A natural question arises here as to whether or not one can, analogously to the classical case, use $\mathrm{S}_{\Phi}$ directly to compute the pullback of $\omega$. For instance, in the case of the universal differential calculus, we can define the pullback of a differential 1-form on $P$ with respect to $\mathrm{s}_{\Phi}$ to be

$$
\mathrm{s}_{\Phi}^{*}\left(\sum_{i} p_{i} d q_{i}\right)=\sum_{i} \mathrm{~s}_{\Phi}\left(p_{i}\right)\left(d \circ \mathrm{s}_{\Phi}\right)\left(q_{i}\right) .
$$

Clearly, since $\mathrm{s}_{\Phi}^{*}\left(\Omega^{1} P\right) \subseteq \Omega^{1} B$ and there exists a non-strong connection on a trivial quantum principal bundle with the universal differential calculus (see Example 2.7), Proposition 2.2 allows one to conclude that, in general, $\mathrm{s}_{\Phi}^{*} \circ \omega$ and $\beta$ given by (13) do not coincide. (Otherwise $\omega$ would always have to be a strong connection form.) Furthermore, even if we assume that $\omega$ is a strong connection form, the direct calculation of $s_{\Phi}^{*} \circ \omega$ shows why, in the noncommutative case, we cannot claim $\mathrm{s}_{\Phi}^{*} \circ \omega=\beta$. An advantage of defining $\beta$ by (13) rather than by $\mathrm{s}_{\Phi}^{*} \circ \omega=\beta$ is that $\beta$ given by formula (13) transforms in a familiar manner under local gauge transformations (see Section 3 and (38) in [9]).

Let us also remark that, assuming the existence of $S^{-1}$, one can define a quantum principal bundle section which is a right, rather than left, $B$-module homomorphism from $P$ to $B$ :

$$
\widetilde{\mathrm{S}}_{\Phi}:=m_{P} \circ\left(\left(\Phi \circ S^{-1}\right) \otimes i d\right) \circ \tau \circ \Delta_{R}
$$

Much as in the case of $\mathrm{s}_{\Phi}$, it is straightforward to check that $\widetilde{\mathrm{s}}_{\Phi}$ is indeed a right $B$-module homomorphism into B. From (28) [9] (see the proof of Corollary 2.4), it is also clear that $\widetilde{\mathrm{S}}_{\Phi}$ satisfies the equation $\widetilde{\mathrm{S}}_{\Phi} \circ \Phi^{-1}=\varepsilon$. This formula and an analogous formula $\mathrm{S}_{\Phi} \circ \Phi=\varepsilon$ for $\mathrm{S}_{\Phi}$ reflect the classical geometry relationship between the section of a principal bundle and the map from the total space to the structure group that are associated to the same trivialization. The pullback of a connection form $\omega$ defined with the help of $\widetilde{\mathrm{s}}_{\Phi}$ has very similar properties to the pullback of $\omega$ defined with the help of $\mathrm{s}_{\Phi}$. Obviously, $\mathrm{s}_{\Phi}$ and $\widetilde{\mathrm{s}}_{\Phi}$ coincide in the classical case.

Due to Proposition 2.2 and Proposition 4.6 [9] we know that every $\beta \in \operatorname{Hom}_{k}\left(A, \Omega^{1} B\right)$ vanishing on $k$ induces a strong connection on a trivial quantum bundle with the universal differential calculus (cf. Section 6.4 in [20]). The quantum Dirac monopole considered in [9] is an example of a strong connection on a quantum bundle with a non-universal differential calculus (for a proof 
of this fact see Corollary 6.4.4 in [6]). In what follows, we present an example of a weak (i.e. nonstrong) connection. This example points out an interesting fact that the noncommutativity alone of the 'total space' $P$ or the 'structure group' $A$ of a quantum principal bundle $P(B, A)$ cannot be held responsible for the weakness of a connection. Nor, for that matter, can we blame the noncocommutativity of $A$.

Example 2.7 Let $2 \mathbb{Z}_{2}$ denote the two-element group represented by 0 and 2 . Also, let $P:=$ $\operatorname{Map}\left(\mathbb{Z}_{4}, k\right)$ and $A:=\operatorname{Map}\left(2 \mathbb{Z}_{2}, k\right)$ be the standard commutative Hopf algebras over $k$ (e.g., see [15] or Section 2.2 in [1]), $\imath: 2 \mathbb{Z}_{2} \hookrightarrow \mathbb{Z}_{4}$ be the inclusion, and

$$
B:=\left\{b \in P \mid \Delta_{R} b:=\left(\left(i d \otimes \imath^{*}\right) \circ \Delta\right)(b)=b \otimes 1\right\}
$$

be the corresponding quantum homogeneous space (see Section 5.1 in [9]). Since

$$
\operatorname{Ker} \imath^{*} \subseteq m_{P} \circ\left(\left(\operatorname{Ker} \imath^{*} \cap B\right) \otimes P\right),
$$

by Lemma 5.2 [9], $\left(P, A,\left(i d \otimes \imath^{*}\right) \circ \Delta, 0,0\right)$ is a quantum principal bundle with the universal differential calculus. Now, let $j: \mathbb{Z}_{4} \rightarrow 2 \mathbb{Z}_{2}$ be the surjection defined by $j(g)=\delta_{2, g}$. Clearly, $\imath \circ j=i d$ and $j\left(h^{-1} g h\right)=h^{-1} j(g) h$ for all $g \in \mathbb{Z}_{4}, h \in 2 \mathbb{Z}_{2}$. (Although $2 \mathbb{Z}_{2}$ and $\mathbb{Z}_{4}$ are additive groups, to emphasize the usefulness of calculations shown in this example even in more general cases, as well as to shorten some formulas, we use the shorter and more abstract multiplicative notation.) Hence, by Proposition 5.3 [9], we have the canonical connection form given by the formula $\omega=(S * d) \circ j^{*}$. Our task is now to show that the connection defined by $\omega$ is weak. Suppose that this is not the case, i.e. that $\left(i d-\Pi^{\omega}\right)(d P) \subseteq \Omega_{\text {shor }}^{1} P$. Then, since it can be calculated that, for all $p \in P$,

$$
\left(i d-\Pi^{\omega}\right)(d p)=d\left(p_{(1)} S\left(\left(\left(j^{*} \circ \imath^{*}\right)\left(p_{(2)}\right)\right)_{(1)}\right)\right) \cdot\left(\left(j^{*} \circ \imath^{*}\right)\left(p_{(2)}\right)\right)_{(2)},
$$

and $\Omega^{1} P$ can be treated as the set of all functions on $\mathbb{Z}_{4} \times \mathbb{Z}_{4}$ vanishing on the diagonal (e.g., see Section 2.6 in $\left[14\right.$ ), we can conclude that, for any $p \in P, g, r \in \mathbb{Z}_{4}, h \in 2 \mathbb{Z}_{2}$,

$$
\begin{gathered}
\left(d\left(p_{(1)} S\left(\left(\left(j^{*} \circ \imath^{*}\right)\left(p_{(2)}\right)\right)_{(1)}\right)\right) \cdot\left(\left(j^{*} \circ \imath^{*}\right)\left(p_{(2)}\right)\right)_{(2)}\right)(g h, r) \\
=\left(d\left(p_{(1)} S\left(\left(\left(j^{*} \circ \imath^{*}\right)\left(p_{(2)}\right)\right)_{(1)}\right)\right) \cdot\left(\left(j^{*} \circ \imath^{*}\right)\left(p_{(2)}\right)\right)_{(2)}\right)(g, r), \\
\text { i.e. } p(r)-p\left(g h j\left(h^{-1} g^{-1} r\right)\right)=p(r)-p\left(g j\left(g^{-1} r\right)\right) .
\end{gathered}
$$

In particular, it implies that

$$
\forall h \in 2 \mathbb{Z}_{2}, g \in \mathbb{Z}_{4}: j(h g)=h j(g)
$$

(cf. Lemma 5.5.5 in [6]). But $h=2, g=1$ do not verify that equality and therefore we have a contradiction proving that $\Pi^{\omega}$ is a weak connection.

Alternatively, since the pullback of the map $\widetilde{\Phi}: \mathbb{Z}_{4} \rightarrow 2 \mathbb{Z}_{2}$ given by

$$
\widetilde{\Phi}: g \longmapsto \begin{cases}0 & \text { for } g \leq 1 \\ 2 & \text { otherwise }\end{cases}
$$

is a trivialization of the quantum bundle $P(B, A)$ (see the Definition 1.4), one can prove that $\Pi^{\omega}$ is a weak connection by analyzing the map $\beta$ associated to it (see (13)) and using Proposition 2.2. Note also that the trivial connection (see Example 4.5 in [9]) induced by the trivialization $\widetilde{\Phi}^{*}$ is, by Proposition 2.2, strong. 
Our next example is concerned with a construction of both strong and non-strong connections

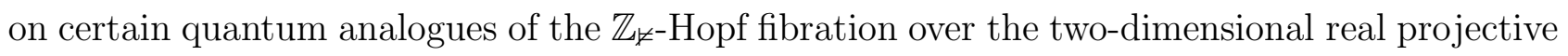
space $\left(S^{2} \rightarrow R P^{2}\right)$.

Example 2.8 Let $P_{F}$ be a unital free *-algebra over $\mathbb{C}$ generated by $\left\{x_{m}\right\}_{m \in\{1,2,3\}}$, (i.e. $P_{F}=\mathbb{C}<\curvearrowleft_{\nVdash} \curvearrowleft_{\sharp}, \curvearrowleft_{\nVdash}, \nVdash>$ ), let $\mathcal{I}$, be a two-sided $*$-ideal of $P_{F}$ generated by

$$
\left\{x_{m}-x_{m}^{*}, \sum_{j} a_{j} x_{j}^{2}-r^{2}\right\}, m, j \in\{1,2,3\}, a_{j}, r \in \mathbb{R}, \partial_{\mathbf{J}}, \backslash>\nvdash,
$$

and let $\mathcal{I}_{\infty}$ be a two-sided $*$-ideal of $P_{F}$ generated by

$$
\begin{aligned}
& \left\{x_{m}-x_{m}^{*}, x_{1}^{2}+x_{2}^{2}+2 \frac{1+q^{4}}{\left(1+q^{2}\right)^{2}} x_{3}^{2}-1, x_{1} x_{2}-x_{2} x_{1}-2 i \frac{1-q^{4}}{\left(1+q^{2}\right)^{2}} x_{3}^{2},\right. \\
& \left.\quad x_{1} x_{3}-\frac{q^{-2}+q^{2}}{2} x_{3} x_{1}-i \frac{q^{2}-q^{-2}}{2} x_{3} x_{2}, x_{2} x_{3}-\frac{q^{-2}+q^{2}}{2} x_{3} x_{2}+i \frac{q^{2}-q^{-2}}{2} x_{3} x_{1}\right\}, \\
& m \in\{1,2,3\}, q \in \mathbb{R}, \nVdash \geq \mid 川>\nvdash .
\end{aligned}
$$

(The second generator of $\mathcal{I}_{\infty}$ resembles the right-hand side of the formula (164) in [26] that describes the metric on the $q$-Minkowski space discussed in Section 7.2 of [26].) The algebras $P_{F} / \mathcal{I}_{1}$ and $P_{F} / \mathcal{I}_{\infty}$ can be regarded as noncommutative two-spheres. Indeed, $P_{F} / \mathcal{I}_{1}$ is 'the most noncommutative two-sphere', and $P_{F} / \mathcal{I}_{\infty}$ corresponds to the equator sphere $(c=\infty)$ given by (7b) in [29] (see Remark 2.13). Obviously, for $q= \pm 1$, the algebra $P_{F} / \mathcal{I}_{\infty}$ corresponds to the usual $S^{2}$. Since both $P_{F} / \mathcal{I}_{1}$ and $P_{F} / \mathcal{I}_{\infty}$ can be used in the same way to construct connections on a noncommutative Hopf fibration, we denote, for the sake of brevity, $P_{F} / \mathcal{I}_{\nu}$ by $P_{\nu}$, where $\nu \in\{0,1\}$. We also put $x_{0 j}=\left[x_{j}\right]_{\mathcal{I}_{1}}, x_{1 j}=\left[x_{j}\right]_{\mathcal{I}_{\infty}}, a_{0 j}=a_{j}, j \in\{1,2,3\}, a_{11}=1, a_{12}=$ $1, a_{13}=2 \frac{1+q^{4}}{\left(1+q^{2}\right)^{2}}, r_{0}=r, r_{1}=1$, and thus define the coefficients $\left\{a_{\nu j}, r_{\nu}\right\}_{\nu \in\{0,1\}, j \in\{1,2,3\}}$. Unless stated otherwise, all the following statements of this example will be valid for any of the two values of $\nu$. The proposition below allows one to turn $P_{\nu}$ into a right $A$-comodule algebra, where $A=\operatorname{Map}\left(\mathbb{Z}_{\not \neq}, \mathbb{C}\right)$.

Proposition 2.9 Let $P_{F}$ and $A$ be as above. Also, let $\Delta_{R}$ be a coaction of $A$ on $P_{F}$ making it a right $A$-comodule algebra. If $\Delta_{R} \circ *=\left(*^{-}\right) \circ \Delta_{R}$, where - denotes the complex conjugation, and

$$
\Delta_{R}: P_{F} \ni x_{j} \longmapsto x_{j} \otimes(1-2 \delta) \in P_{F} \otimes A,
$$

where $\delta$ is the map such that $\delta(-1)=1$ and $\delta(1)=0$, then $\Delta_{R}\left(\mathcal{I}_{\nu}\right) \subseteq \mathcal{I}_{\nu} \otimes \mathcal{A}$.

Proof. Clearly, $\Delta_{R}\left(x_{j}-x_{j}^{*}\right) \in \mathcal{I}_{\nu} \otimes \mathcal{A}$ for any $j \in\{1,2,3\}$. Also, for any $j, l \in\{1,2,3\}$, we have

$$
\Delta_{R}\left(x_{j} x_{l}\right)=\Delta_{R}\left(x_{j}\right) \Delta_{R}\left(x_{l}\right)=x_{j} x_{l} \otimes(1-2 \delta)^{2}=x_{j} x_{l} \otimes 1 .
$$

Hence $\Delta_{R}\left(\mathcal{I}_{\nu}\right) \subseteq \mathcal{I}_{\nu} \otimes \mathcal{A}$, as claimed.

It follows now that a $*$-algebra homomorphism $\Delta_{\nu}: P_{\nu} \rightarrow P_{\nu} \otimes A$ given by the formula $\Delta_{\nu} x_{\nu j}=$ $x_{\nu j} \otimes(1-2 \delta)$ makes $P_{\nu}$ a right $A$-comodule algebra.

Proposition 2.10 Let $P_{\nu}$ be a right $A$-comodule algebra as above. Then $\left(P_{\nu}, A, \Delta_{\nu}, 0,0\right)$ is a quantum principal bundle with the universal differential calculus. 
Proof. By Proposition 1.6, it suffices to show that the canonical map $\mathrm{T}_{B}: P_{\nu} \otimes_{B_{\nu}} P_{\nu} \rightarrow P_{\nu} \otimes A$, where $B_{\nu}:=P_{\nu}{ }^{c o A}$, is bijective. First, however, let us prove the following

Lemma 2.11 Let $B_{\nu}$ be as above. Then $B_{\nu}$ is the space spanned by monomials from $P_{\nu}$ whose total degree is even, i.e.

$$
B_{\nu}=\left\{\sum_{k \geq 1} \sum_{i_{1}, \ldots, i_{2 k}} a_{i_{1} \ldots i_{2 k}} x_{\nu i_{1}} \cdots x_{\nu i_{2 k}} \in P_{\nu} \mid a_{i_{1} \ldots i_{2 k}} \in \mathbb{C}, \beth_{\nVdash}, \ldots, \beth_{\sharp\urcorner} \in\{\nVdash, \not \models, \nVdash\}\right\} .
$$

Proof. To simplify notation, let us denote the right hand side of (23) by $\widetilde{B_{\nu}}$. Thanks to (22), it is clear that every element of $\widetilde{B_{\nu}}$ is right coinvariant. It is also clear that every element of $P_{\nu}$ can be written as $b_{0}+\sum_{j=1}^{3} b_{j} x_{\nu j}$ for some $\left\{b_{l}\right\}_{l \in\{0, \ldots, 3\}} \subseteq \widetilde{B_{\nu}}$. (Observe that any number $c \in \mathbb{C}$ can be expressed as $c=c r_{\nu}^{-2} \sum_{i=1}^{3} a_{\nu i} x_{\nu i}^{2} \in \widetilde{B_{\nu}}$.) Furthermore, since

$$
\Delta_{\nu} p-p \otimes 1=b_{0} \otimes 1+\sum_{j=1}^{3}\left(b_{j} x_{\nu j} \otimes 1-2 b_{j} x_{\nu j} \otimes \delta\right)-b_{0} \otimes 1-\sum_{j=1}^{3} b_{j} x_{\nu j} \otimes 1=-2\left(\sum_{j=1}^{3} b_{j} x_{\nu j}\right) \otimes \delta
$$

we can conclude that $\Delta_{\nu} p=p \otimes 1 \Rightarrow p=b_{0} \in \widetilde{B_{\nu}}$. Hence $B_{\nu}=\widetilde{B_{\nu}}$, as claimed.

Now, consider a left $P_{\nu}$-module map $\widetilde{\mathrm{T}}_{B}: P_{\nu} \otimes A \rightarrow P_{\nu} \otimes_{B_{\nu}} P_{\nu}$ given by the formula:

$$
\widetilde{\mathrm{T}}_{B}(1 \otimes a) \longmapsto \begin{cases}1 \otimes_{B_{\nu}} 1 & \text { for } a=1 \\ \frac{1}{2}\left(1 \otimes_{B_{\nu}} 1-r_{\nu}^{-2} \sum_{i=1}^{3} a_{\nu i} x_{\nu i} \otimes_{B_{\nu}} x_{\nu i}\right) & \text { for } a=\delta\end{cases}
$$

Recall that every element of $P_{\nu}$ can be written as $b_{0}+\sum_{j=1}^{3} b_{j} x_{\nu j}$ for some $\left\{b_{l}\right\}_{l \in\{0, \ldots, 3\}} \subseteq B_{\nu}$. Therefore, since $\widetilde{\mathrm{T}}_{B} \circ \mathrm{T}_{B}$ is a left $P_{\nu}$-module map, it suffices to check that

$$
\left(\widetilde{\mathrm{T}}_{B} \circ \mathrm{T}_{B}\right)\left(1 \otimes_{B_{\nu}}\left(b_{0}+\sum_{j=1}^{3} b_{j} x_{\nu j}\right)\right)=1 \otimes_{B_{\nu}}\left(b_{0}+\sum_{j=1}^{3} b_{j} x_{\nu j}\right)
$$

for arbitrary $\left\{b_{l}\right\}_{l \in\{0, \ldots, 3\}} \subseteq B_{\nu}$. With the help of Lemma 2.11, we have

$$
\begin{aligned}
& \left(\widetilde{\mathrm{T}}_{B} \circ \mathrm{T}_{B}\right)\left(1 \otimes_{B_{\nu}}\left(b_{0}+\sum_{j=1}^{3} b_{j} x_{\nu j}\right)\right) \\
& =b_{0}\left(\widetilde{\mathrm{T}}_{B} \circ \mathrm{T}_{B}\right)\left(1 \otimes_{B_{\nu}} 1\right)+\sum_{j=1}^{3} b_{j}\left(\widetilde{\mathrm{T}}_{B} \circ \mathrm{T}_{B}\right)\left(1 \otimes_{B_{\nu}} x_{\nu j}\right) \\
& =b_{0} \otimes_{B_{\nu}} 1+\sum_{j=1}^{3} b_{j} \widetilde{\mathrm{T}}_{B}\left(x_{\nu j} \otimes 1-2 x_{\nu j} \otimes \delta\right) \\
& =b_{0} \otimes_{B_{\nu}} 1+\sum_{j=1}^{3} b_{j}\left(x_{\nu j} \otimes_{B_{\nu}} 1-2 x_{\nu j} \widetilde{\mathrm{T}}_{B}(1 \otimes \delta)\right) \\
& =b_{0} \otimes_{B_{\nu}} 1+\sum_{j=1}^{3}\left(b_{j} x_{\nu j} \otimes_{B_{\nu}} 1-b_{j} x_{\nu j} \otimes_{B_{\nu}} 1+r_{\nu}^{-2} \sum_{i=1}^{3}\left(a_{\nu i} b_{j} x_{\nu j} x_{\nu i} \otimes_{B_{\nu}} x_{\nu i}\right)\right) \\
& =1 \otimes_{B_{\nu}} b_{0}+\sum_{j=1}^{3} \sum_{i=1}^{3} r_{\nu}^{-2} a_{\nu i} \otimes_{B_{\nu}} b_{j} x_{\nu j} x_{\nu i}^{2} \\
& =1 \otimes_{B_{\nu}}\left(b_{0}+\sum_{j=1}^{3} b_{j} x_{\nu j} \sum_{i=1}^{3} r_{\nu}^{-2} a_{\nu i} x_{\nu i}^{2}\right) \\
& =1 \otimes_{B_{\nu}}\left(b_{0}+\sum_{j=1}^{3} b_{j} x_{\nu j}\right) .
\end{aligned}
$$

Thus we have shown that $\widetilde{\mathrm{T}}_{B} \circ \mathrm{T}_{B}=i d$. Furthermore, it is straightforward to verify that $\mathrm{T}_{B} \circ \widetilde{\mathrm{T}}_{B}=i d$. Hence $\widetilde{\mathrm{T}}_{B}$ is the inverse of $\mathrm{T}_{B}$ and consequently $\mathrm{T}_{B}$ is bijective, as needed. 
Remark 2.12 Note that, since $\operatorname{Map}\left(\mathbb{Z}_{\sharp}, \mathbb{C}\right)$ is finite dimensional, the injectivity of $\mathrm{T}_{B}$ follows immediately from its surjectivity and Theorem 1.3 in [32].

Remark 2.13 Recall that a classical point of an algebra $B$ over a field $k$ is defined as an algebra homomorphism from $B$ to $k$. For $q \neq \pm 1$, the space of all classical points of $P_{1}$ is parameterized by all pairs $(x, y) \in \mathbb{R}^{\nvdash}$ subject to the relation $x^{2}+y^{2}=r_{1}=1$. Any such pair yields an algebra homomorphism $f: P_{1} \rightarrow \mathbb{C}$ via the formulas $f\left(x_{11}\right)=x, f\left(x_{12}\right)=y, f\left(x_{13}\right)=0$. It is clear that the classical 'subspace' of $P_{1}$ is precisely its equator. Hence the name 'equator sphere'. (To see the correspondence between $P_{1}$ and the $C^{*}$-algebra defined by (7b) 29], put $\mu=q$ and

$$
x_{11}=\frac{i}{2}\left(B^{*}-B\right), x_{12}=-\frac{1}{2}\left(B^{*}+B\right), x_{13}=\frac{-1-q^{2}}{2} A ;
$$

cf. the beginning of Section 7 in [29].) Now, note that the quantum sphere employed in Section 5.2 of [9] ( $c=0$ in [29]) can be, in the same manner, regarded as a north pole sphere. On the other hand, the quantum principal bundles considered in Proposition 2.10 were constructed to generalize the usual Hopf fibration $S^{2} \rightarrow R P^{2}$ (set $q= \pm 1$ in the bundle $\left(P_{1}, A, \Delta_{1}, 0,0\right)$ ) where $\mathbb{Z}_{\not}$ moves the points on the sphere to their antipodal counterparts (see p.69 in [37]). On the north pole sphere used in [9], there is no other classical point to which the north pole could be moved under the free action of $\mathbb{Z}_{\not k}$. This is why, in order to deform the Hopf fibration $S^{2} \rightarrow R P^{2}$, we used here the equator sphere instead. [f

Proposition 2.14 Let $P_{\nu}\left(B_{\nu}, A\right)$ be a quantum principal bundle as in Proposition 2.10 and Lemma 2.11. Also, let $\omega \in \operatorname{Hom}_{\mathbb{C}}\left(A, \Omega^{1} P_{\nu}\right)$ be a homomorphism defined by the formula

$$
\omega(a)= \begin{cases}0 & \text { for } a=1 \\ -\frac{1}{2 r_{\nu}^{2}} \sum_{i=1}^{3} a_{\nu i} x_{\nu i} d x_{\nu i} & \text { for } a=\delta\end{cases}
$$

Then $\omega$ is a strong connection form on $P_{\nu}\left(B_{\nu}, A\right)$.

Proof. To prove that $\omega$ is a connection form it suffices to verify that $(\mathrm{T} \circ \omega) \delta=1 \otimes \delta$. (Other conditions of Definition 1.8 are immediately satisfied.) We have

$$
\begin{aligned}
(\mathrm{T} \circ \omega) \delta & =-\frac{1}{2 r_{\nu}^{2}} \sum_{i=1}^{3} a_{\nu i} x_{\nu i} \mathrm{~T}_{R}\left(1 \otimes x_{\nu i}-x_{\nu i} \otimes 1\right) \\
& =-\frac{1}{2 r_{\nu}^{2}} \sum_{i=1}^{3} a_{\nu i} x_{\nu i}\left(x_{\nu i} \otimes 1-2 x_{\nu i} \otimes \delta-x_{\nu i} \otimes 1\right) \\
& =1 \otimes \delta .
\end{aligned}
$$

\footnotetext{
${ }^{2}$ I am grateful to Stanisław Zakrzewski for explaining these things to me.
} 
Hence $\omega$ is indeed a connection form. Our next step is to show that $\omega$ is strong (see Defini-

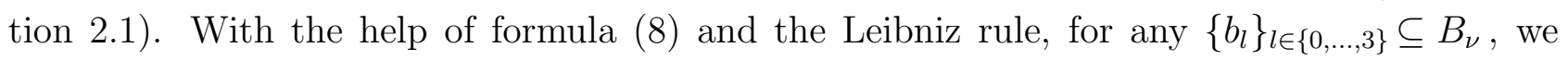
have

$$
\begin{aligned}
\left(\Pi^{\omega} \circ d\right)\left(b_{0}+\sum_{j=1}^{3} b_{j} x_{\nu j}\right) & =-2 \sum_{j=1}^{3} b_{j} x_{\nu j} \omega(\delta) \\
& =r_{\nu}^{-2} \sum_{j=1}^{3} \sum_{i=1}^{3} a_{\nu i} b_{j} x_{\nu j} x_{\nu i} d x_{\nu i} \\
& =r_{\nu}^{-2} \sum_{j=1}^{3} \sum_{i=1}^{3} a_{\nu i} b_{j}\left(d\left(x_{\nu j} x_{\nu i}^{2}\right)-d\left(x_{\nu j} x_{\nu i}\right) \cdot x_{\nu i}\right) \\
& =\sum_{j=1}^{3} b_{j} d x_{\nu j}-r_{\nu}^{-2} \sum_{i, j \in\{1,2,3\}} a_{\nu i} b_{j} d\left(x_{\nu j} x_{\nu i}\right) \cdot x_{\nu i} .
\end{aligned}
$$

Applying the Leibniz rule again, we obtain

$$
\left(i d-\Pi^{\omega}\right)\left(d\left(b_{0}+\sum_{j=1}^{3} b_{j} x_{\nu j}\right)\right)=d b_{0}+\sum_{j=1}^{3} d b_{j} \cdot x_{\nu j}+r_{\nu}^{-2} \sum_{i, j \in\{1,2,3\}} a_{\nu i} b_{j} d\left(x_{\nu j} x_{\nu i}\right) . x_{\nu i} \in \Omega_{s h o r}^{1} P_{\nu} .
$$

Taking advantage of the fact that any $p \in P_{\nu}$ can be expressed as $b_{0}+\sum_{j=1}^{3} b_{j} x_{\nu j}$ for some

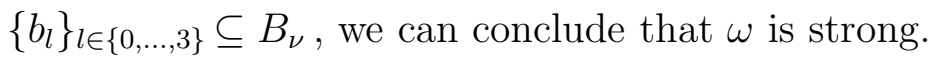

Proposition 2.15 Let $P_{\nu}\left(B_{\nu}, A\right)$ and $\omega$ be as in the proposition above. A homomorphism $\widetilde{\omega} \in \operatorname{Hom}_{\mathbb{C}}\left(A, \Omega^{1} P_{\nu}\right)$ defined by the formula

$$
\widetilde{\omega}(a)= \begin{cases}0 & \text { for } a=1 \\ \omega(\delta)+d x_{\nu l}^{2} & \text { for } a=\delta,\end{cases}
$$

where $l \in\{1,2,3\}$, is a connection 1-form of a connection that is not strong.

Proof. Let $l$ be any fixed element of $\{1,2,3\}$. Since $\Delta_{\mathcal{R}}\left(d x_{\nu l}^{2}\right)=d x_{\nu l}^{2} \otimes 1$ and $\mathrm{T}\left(d x_{\nu l}^{2}\right)=0$, it is clear that $\widetilde{\omega}$ is a connection 1 -form. To prove that $\Pi^{\tilde{\omega}}$ is not a strong connection, we will demonstrate that $\left(i d-\Pi^{\tilde{\omega}}\right)\left(d x_{\nu l}\right) \notin \Omega_{\text {shor }}^{1} P_{\nu}$. With the help of formula (8), we have:

$$
\left(i d-\Pi^{\tilde{\omega}}\right)\left(d x_{\nu l}\right)=d x_{\nu l}+2 x_{\nu l} \widetilde{\omega}(\delta)=d x_{\nu l}+2 x_{\nu l} \omega(\delta)+2 x_{\nu l} d x_{\nu l}^{2}=\left(i d-\Pi^{\omega}\right)\left(d x_{\nu l}\right)+2 x_{\nu l} d x_{\nu l}^{2}
$$

Therefore, as $\Pi^{\omega}$ is a strong connection, it is enough to show that $x_{\nu l} d x_{\nu l}^{2} \quad k \Omega_{s h o r}^{1} P_{\nu}$. To this end, let us put $B_{\nu}^{c}:=\left\{\sum_{j=1}^{3} b_{j} . x_{\nu j} \in P_{\nu} \mid b_{j} \in B_{\nu}, j \in\{1,2,3\}\right\}$. Clearly, $P_{\nu}=B_{\nu}+B_{\nu}^{c}$. An argument similar to that used in the proof of Lemma 2.11 shows that $B_{\nu} \cap B_{\nu}^{c}=0$. It follows now that $P_{\nu}=B_{\nu} \oplus B_{\nu}^{c}$. As we consider here the universal calculus on $P_{\nu}$, we have an isomorphism $\psi: \Omega^{1} P_{\nu} \ni d p . u \mapsto[p]_{\mathbb{C}} \otimes u \in P_{\nu} / \mathbb{C} \otimes P_{\nu}$. Furthermore, we have

$$
\begin{aligned}
\Omega^{1} P_{\nu} & =\psi^{-1}\left(\psi\left(\Omega^{1} P_{\nu}\right)\right) \\
& =\psi^{-1}\left(B_{\nu} / \mathbb{C} \otimes P_{\nu}\right) \oplus \psi^{-\nVdash}\left(\left(\mathbb{B}_{\nu} \oplus \mathbb{C}\right) / \mathbb{C} \otimes P_{\nu}\right) \\
& =d B_{\nu} \cdot P_{\nu} \oplus d\left(B_{\nu}^{c} \oplus \mathbb{C}\right) \cdot P_{\nu} \\
& =\Omega^{1} B_{\nu} \cdot P_{\nu} \oplus d\left(B_{\nu}^{c}\right) \cdot P_{\nu} .
\end{aligned}
$$


On the other hand, taking into account the isomorphism $\Omega^{1} P_{\nu} \cong P_{\nu} \otimes\left(P_{\nu} / \mathbb{C}\right)$, one can show (with some help of the representation presented in Point III.(a) of Proposition 4 in [29] and formulas (24)) that $x_{\nu l} d x_{\nu l}^{2} \neq 0$. Therefore, since $x_{\nu l} d x_{\nu l}^{2}=d x_{\nu l}^{3}-d x_{\nu l} . x_{\nu l}^{2} \in d\left(B_{\nu}^{c}\right) . P_{\nu}$, we can conclude, by virtue of (25), that $x_{\nu l} d x_{\nu l}^{2} \notin \Omega_{\text {shor }}^{1} P_{\nu}$, as desired.

Remark 2.16 The strong connection form $\omega$ defined in Proposition 2.14 is non-trivial. Indeed, taking (8) into account, one can see that

$$
\Pi^{\omega}\left(d x_{\nu l}\right)=-2 x_{\nu l} \omega(\delta)=r_{\nu}^{-2} \sum_{i=1}^{3} a_{\nu i} x_{\nu l} x_{\nu i} d x_{\nu i}
$$

where, as before, $l$ is any fixed element of $\{1,2,3\}$. On the other hand, it can be checked that $\left\{\left[x_{\nu i}\right]_{\mathbb{C}}\right\}_{i \in\{1,2,3\}}$ are linearly independent and that $x_{\nu l}^{2} \neq 0$. (Again, one can take advantage of the representation presented in Point III.(a) of Proposition 4 in [29] and formulas (24).) Hence, with the help of an isomorphism $\Omega^{1} P_{\nu} \cong P_{\nu} \otimes\left(P_{\nu} / \mathbb{C}\right)$, it follows that $\Pi^{\omega}\left(d x_{\nu l}\right) \neq 0$. Consequently, the space of vertical forms (i.e. $\operatorname{Im} \Pi^{\omega}$ ) is non-zero.

We end our display of examples with a strict monoidal category (see Section 6.1 in [33]) example of a weak connection.

Example 2.17 Similarly to Example 2.7, this example is concerned with a translation of the concept of the canonical connection on a homogeneous space to a different set-up. Only this time, the groups employed are neither Abelian, nor finite. The former makes our bundle look more interesting, the latter forces us to replace the algebraic tensor product by the appropriate dual of the Cartesian product. More precisely, let $\mathfrak{M}$ be the image of the category of sets under the contravariant functor $\operatorname{Map}(\cdot, k)$. The tensor product $\otimes_{\mathfrak{M}}$ defined by

$$
\operatorname{Map}(X, k) \otimes_{\mathfrak{M}} \operatorname{Map}(Y, k)=\operatorname{Map}(X \times Y, k)
$$

makes $\mathfrak{M}$ a strict monoidal category. Moreover, if $X$ is a group, then $\operatorname{Map}(X, k)$ is an $\mathfrak{M}$-Hopf algebra, where the definition of an $\mathfrak{M}$-Hopf algebra is the same as that of a Hopf algebra but with the tensor product taken to be $\otimes_{\mathfrak{M}}$. In what follows we define several gauge theoretic notions in the setting of the category $\mathfrak{M}$ :

1. $\Omega_{\mathfrak{M}}^{1} X:=\{F \in \operatorname{Map}(X \times X, k) \mid \forall x \in X: F(x, x)=0\}$ (Map $(X, k)$-bimodule of $\mathfrak{M}$-differential 1 -forms)

2. $\Delta_{\mathfrak{M}-\mathfrak{R}}:=R^{*}$ (right coaction), where $R: X \times G \rightarrow X$ is a right free action of the group $G$ on $X$ (e.g., see p.55 in [37]; $G$ will denote a group and $R$ its right free action throughout the rest of this example)

3. $\Delta_{\mathfrak{M}-\mathcal{R}}: \Omega_{\mathfrak{M}}^{1} X \rightarrow\{K \in \operatorname{Map}(X \times X \times G, k) \mid \forall x \in X, g \in G: K(x, x, g)=0\}$ (right coaction on $\mathfrak{M}$-differential 1-forms), where $\forall F \in \Omega_{\mathfrak{M}}^{1} X, x, y \in X, g \in G:\left(\Delta_{\mathfrak{M}-\mathcal{R}} F\right)(x, y, g):=$ $F(R(x, g), R(y, g))$ 
4. A triple $\left(\operatorname{Map}(X, k), \operatorname{Map}(G, k), \Delta_{\mathfrak{M}-\mathfrak{R}}\right)$ is called an $\mathfrak{M}$-principal bundle and, for simplicity, denoted by $(X, G, R)$.

5. $\Omega_{\mathfrak{M}-\mathfrak{h o r}}^{1} X:=\left\{F \in \Omega_{\mathfrak{M}}^{1} X \mid \forall x \in X, g \in G: F(R(x, g), x)=0\right\}$ (horizontal $\mathfrak{M}$-differential 1 -forms)

6. $B:=\operatorname{Map}(X / G, k)$ (base space of $(X, G, R))$

7. $\Omega_{\mathfrak{M}-\mathfrak{s h o r}}^{1} X:=\left\{F \in \Omega_{\mathfrak{M}-\mathfrak{h o r}}^{1} X \mid \forall x, y \in X, g \in G: F(R(x, g), y)=F(x, y)\right\}$ (strongly horizontal $\mathfrak{M}$-differential 1-forms)

8. Let $(X, G, R)$ be an $\mathfrak{M}$-principal bundle and let

$$
\widetilde{\Pi}:=\widetilde{\Pi}_{1} \times \widetilde{\Pi}_{2} \in \operatorname{Map}(X \times X, X \times X)
$$

be an idempotent satisfying the following conditions:

a) $\forall x, y \in X: \widetilde{\Pi}_{1}(x, y)=x$,

b) $\forall F \in \Omega_{\mathfrak{M}}^{1} X: F \circ \widetilde{\Pi}=0 \Leftrightarrow F \in \Omega_{\mathfrak{M}-\mathfrak{h o r}}^{1} X$,

c) $\forall x, y \in X, g \in G: \widetilde{\Pi}_{2}(R(x, g), R(y, g))=R\left(\widetilde{\Pi}_{2}(x, y), g\right)$.

Then $\widetilde{\Pi}^{*}$ is called an $\mathfrak{M}$-connection and is denoted by $\Pi_{\mathfrak{M}}$.

9. An $\mathfrak{M}$-connection $\Pi_{\mathfrak{M}}$ is called a strong $\mathfrak{M}$-connection iff

$$
\forall x, y \in X, g \in G: \widetilde{\Pi}_{2}(R(x, g), y)=\widetilde{\Pi}_{2}(x, y) .
$$

The above definitions were constructed so that the $\mathfrak{M}$-objects thus defined become the corresponding 'quantum objects' (the universal differential calculus assumed) when both $X$ and $G$ are finite and the $\mathfrak{M}$-tensor product is the same as the algebraic tensor product. In particular, one can rethink and equivalently describe Example 2.7 in $\mathfrak{M}$-terms. Doing so blurs the view of general principles making that example work, but it allows one to have a better insight into its concrete mathematical fabric.

Clearly, if $H$ is a subgroup of $G$ acting on $G$ on the right by the group multiplication (let us denote this action by $\left.R_{G}\right)$, then $\left(G, H, R_{G}\right)$ is an $\mathfrak{M}$-principal bundle. Furthermore, any surjection $j: G \rightarrow H$ satisfying $j \circ \imath=i d$, where $\imath: H \hookrightarrow G$ is the inclusion, and

$$
\forall g \in G, h \in H: j\left(h^{-1} g h\right)=h^{-1} j(g) h
$$

yields an $\mathfrak{M}$-connection on $\left(G, H, R_{G}\right)$. Indeed, let $\widetilde{\Pi}^{j}(g, r):=\left(g, g j\left(g^{-1} r\right)\right)$, for any $g, r \in G$. Then, for all $g, r \in G, h \in H$, we have:

1. $\left(\widetilde{\Pi}^{j}\right)^{2}(g, r)=\left(g, g j\left(g^{-1} g j\left(g^{-1} r\right)\right)\right)=\left(g, g j\left(g^{-1} r\right)\right)=\widetilde{\Pi}^{j}(g, r)$, where the middle equality is implied by the formula $j \circ \imath=i d$.

2. $\widetilde{\Pi}_{1}^{j}(g, r)=g$ (obvious).

3. $F \in \Omega_{\mathfrak{M}-\mathfrak{h o r}}^{1} G \Rightarrow F \circ \widetilde{\Pi}_{j}=0$ (obvious). 
4. For any $F \in \operatorname{Map}(G \times G, k)$, the implication

$$
F \circ \widetilde{\Pi}^{j}=0 \Rightarrow F \in \Omega_{\mathfrak{M}-\mathfrak{h o r}}^{1} G
$$

is a consequence of the fact that $\widetilde{\Pi}_{j}$ restricted to $\bigcup_{g \in G}(g, g H)$ coincides with the inclusion of $\bigcup_{g \in G}(g, g H)$ in $G \times G$.

5. $\widetilde{\Pi}_{2}^{j}(g h, r h)=g h j\left(h^{-1} g^{-1} r h\right)=g j\left(g^{-1} r\right) h=\widetilde{\Pi}_{2}^{j}(g, r) h$, where the middle step follows from (26).

Hence, $\left(\widetilde{\Pi}^{j}\right)^{*}$ is an $\mathfrak{M}$-connection. Moreover, much as in Example 2.7, if $\left(\widetilde{\Pi}^{j}\right)^{*}$ is a strong $\mathfrak{M}$-connection, then, for all $g \in G, h \in H, h j(g)=j(h g)$.

Next, we proceed to consider a special case of $G$ and $H$. To do so, first we need a definition of an algebraic formal group:

Definition 2.18 (cf. [25] and Appendix A in [15]) Let $\mathfrak{g}$ be a finite dimensional Lie algebra $(\operatorname{dim} \mathfrak{g}=\mathfrak{n})$, and let $\left\{E^{\nu}\right\}_{\nu \in\{1, \cdots, n\}}$ be a basis of $\mathfrak{g}$. Also, let $F$ be the formal group law (see, e.g., Section 9.1 and Section 1.1 in 25]) given by the Baker-Campbell-Hausdorff formula determined by $\mathfrak{g}$ and the basis $\left\{E^{\nu}\right\}_{\nu \in\{1, \cdots, n\}}$ (see Appendix $A$ in $[15]$ ), so that to every pair of $n$-tuples of formal power series in a finite number of variables with no free term we can assign another such $n$-tuple, i.e.

$$
\begin{aligned}
& F\left(\left(p_{1}\left(t_{1}, \cdots, t_{l}\right), \cdots, p_{n}\left(t_{1}, \cdots, t_{l}\right)\right),\left(q_{1}\left(s_{1}, \cdots, s_{m}\right), \cdots, q_{n}\left(s_{1}, \cdots, s_{m}\right)\right)\right) \\
& =\left(r_{1}\left(t_{1}, \cdots, t_{l}, s_{1}, \cdots, s_{m}\right), \cdots, r_{n}\left(t_{1}, \cdots, t_{l}, s_{1}, \cdots, s_{m}\right)\right) .
\end{aligned}
$$

Symbolically, we will write $\left(p_{1}\left(t_{1}, \cdots, t_{l}\right), \cdots, p_{n}\left(t_{1}, \cdots, t_{l}\right)\right)$ as $\exp \left(p_{\nu} E^{\nu}\right)$ and $F\left(\exp \left(p_{\nu} E^{\nu}\right), \exp \left(q_{\mu} E^{\mu}\right)\right)$ as $\exp \left(p_{\nu} E^{\nu}\right) \exp \left(p_{\nu} E^{\nu}\right)$. (The Einstein convention of summation over repeating indices is assumed here and throughout the rest of this example.) The group generated with the use of $F$ by the $n$-tuples $\left\{\exp \left(t_{m} X\right)\right\}_{m \in \mathbb{N}, X \in \mathfrak{g}}$, where $\left\{t_{m}\right\}_{m \in \mathbb{N}}$ are formal power series in one variable with all but the linear coefficients vanishing, is called the algebraic formal group associated to $\mathfrak{g}$ and is denoted by $E_{\mathfrak{g}}$.

Now, let $G=E_{\mathfrak{s l}(2, \mathbb{C})}, H=E_{\mathfrak{s u}(2)}$ and let $j: E_{\mathfrak{s l}(2, \mathbb{C})} \rightarrow E_{\mathfrak{s u}(2)}$ be the surjection defined by $j\left(\exp \left(p_{l} E^{l}+p_{\mu} E^{\mu}\right)\right)=\exp \left(p_{l} E^{l}\right)$, where $\left\{E^{l}\right\}_{l \in\{1,2,3\}}$ is a fixed basis of $\mathfrak{s u}(2)$, and $\left\{E^{\mu}\right\}_{\mu \in\{1,2,3\}}$ is a fixed basis of $i \mathfrak{s u}(2)$. It is clear that $\left(E_{\mathfrak{s l}(2, \mathbb{C})}, E_{\mathfrak{s u}(2)}, R_{E_{\mathfrak{S l}(2, \mathbb{C})}}\right)$ is an $\mathfrak{M}$-principal bundle, and $j \circ \imath=i d$. Thus, to see that $j$ induces an $\mathfrak{M}$-connection, it suffices to prove the following:

Lemma 2.19 $\forall g \in E_{\mathfrak{s l}(2, \mathbb{C})}, h \in E_{\mathfrak{s u}(2)}: j\left(h^{-1} g h\right)=h^{-1} j(g) h$

Proof. Since the formal power series determining elements of $E_{\mathfrak{s l}(2, \mathbb{C})}$ are generated by the Baker-Campbell-Hausdorff formula, we know that

$$
\begin{array}{r}
\forall g \in E_{\mathfrak{s l}(2, \mathbb{C})} \exists \varepsilon_{g}>0, n \in \mathbb{N} \forall \bar{x} \in\left\{\left(z_{1}, \cdots, z_{n}\right) \in \mathbb{R}^{\ltimes} \mid \digamma_{\nVdash}^{\not \not}+\cdots+\digamma_{\ltimes}^{\not \nvdash}<\varepsilon_{丂}^{\not \nvdash}\right\}: \\
V_{\bar{x}}(g):=\exp \left(p_{l}(\bar{x}) E^{l}+p_{\mu}(\bar{x}) E^{\mu}\right) \in S L(2, \mathbb{C}) .
\end{array}
$$


This means that the formal power series $\left\{p_{l}, p_{\mu}\right\}_{l, \mu \in\{1,2,3\}}$ defining $g$ are convergent when evaluated at any $\bar{x}$ in an $\varepsilon$-neighborhood of $0 \in \mathbb{R}^{n}$. Now, let $g$ be an arbitrary element of $E_{\mathfrak{s l}(2, \mathbb{C})}$ and $h$ be any element of $E_{\mathfrak{s u}(2)}$ of the form $h=\exp (t X)$, where $X \in \mathfrak{s u}(2)$ and $t$ is understood as a formal power series in one variable. Also, let $\varepsilon:=\min \left\{\varepsilon_{h^{-1} j(g) h}, \varepsilon_{j\left(h^{-1} g h\right)}\right\}, \bar{y}:=(\bar{t}, \bar{x}) \in \mathbb{R}^{n+1}$ and $\widetilde{j}$ be the map defined on all elements of $S L(2, \mathbb{C})$ of the form $\exp \left(t_{l} E^{l}+t_{\mu} E^{\mu}\right), \forall l, \mu: t_{l}, t_{\mu} \in \mathbb{R}$, by the formula

$$
\widetilde{j}\left(\exp \left(t_{l} E^{l}+t_{\mu} E^{\mu}\right)\right)=\exp \left(t_{l} E^{l}\right) .
$$

Then, using the fact that the splitting $\mathfrak{s l}(2, \mathbb{C})=\mathfrak{s u}(2) \oplus i \mathfrak{s u}(2)$ is ad-invariant, for every $\bar{y}$ of length smaller than $\varepsilon$, we have

$$
\begin{aligned}
& \left(V_{\bar{y}} \circ j\right)\left(\exp (-t X) \exp \left(p_{l} E^{l}+p_{\mu} E^{\mu}\right) \exp (t X)\right) \\
& =\left(\widetilde{j} \circ V_{\bar{y}}\right)\left(\exp (-t X) \exp \left(p_{l} E^{l}+p_{\mu} E^{\mu}\right) \exp (t X)\right) \\
& =\widetilde{j}\left(\exp (-\bar{t} X) \exp \left(p_{l}(\bar{x}) E^{l}+p_{\mu}(\bar{x}) E^{\mu}\right) \exp (\bar{t} X)\right) \\
& =\widetilde{j}\left(\exp \left(p_{l}(\bar{x}) \exp (-\bar{t} X) E^{l} \exp (\bar{t} X)+p_{\mu}(\bar{x}) \exp (-\bar{t} X) E^{\mu} \exp (\bar{t} X)\right)\right) \\
& =\exp \left(p_{l}(\bar{x}) \exp (-\bar{t} X) E^{l} \exp (\bar{t} X)\right) \\
& =\exp (-\bar{t} X) \exp \left(p_{l}(\bar{x}) E^{l}\right) \exp (\bar{t} X) \\
& =V_{\bar{y}}\left(\exp (-t X) \exp \left(p_{l} E^{l}\right) \exp (t X)\right) \\
& =V_{\bar{y}}\left(\exp (-t X) j\left(\exp \left(p_{l} E^{l}+p_{\mu} E^{\mu}\right)\right) \exp (t X)\right)
\end{aligned}
$$

Thus, the formal power series defining $j\left(h^{-1} g h\right)$ and $h^{-1} j(g) h$ coincide when evaluated on an open neighborhood of $0 \in \mathbb{R}^{n+1}$, and hence are identical. To end the proof, we need to note that $a d: E_{\mathfrak{s u}(2)} \rightarrow \operatorname{Aut}\left(E_{\mathfrak{s l}(2, \mathbb{C})}\right)$ is a homomorphism and, since every element of $E_{\mathfrak{s u}(2)}$ is generated by elements of the form $\exp (t X)$, the formula $j\left(h^{-1} g h\right)=h^{-1} j(g) h$ is valid for all $g \in E_{\mathfrak{s l}(2, \mathbb{C})}$ and $h \in E_{\mathfrak{s u}(2)}$, as claimed.

Finally, since $h=\exp (t Y), g=\exp (s Z)$, where $Y:=\left(\begin{array}{cc}0 & 1 \\ -1 & 0\end{array}\right), Z:=\left(\begin{array}{cc}1 & 0 \\ 0 & -1\end{array}\right)$, do not satisfy $j(h g)=h j(g)$, the $j$-induced $\mathfrak{M}$-connection is non-strong, as desired.f

It seems proper to mention at this point that it would be interesting to see to what extent gauge theory on quantum principal bundles can work in some more interesting categories and whether the monoidal reconstruction (see Section 8.2 in [33]) can be extended to reconstruct bundles and connections.

\section{Gauge Transformations}

The next natural step is to determine how strong connections behave under quantum gauge transformations. To do so, we must first define gauge transformations of a quantum principal bundle. One can define the group of quantum gauge transformations as the group of convolution-invertible elements of $\operatorname{Hom}_{k}(A, P)$ which intertwine $\Delta_{R}$ with $a d_{R}$, and satisfy $f(1)=1$. (The same definition is considered in Proposition 5.2 of [0].) Then one can define

\footnotetext{
${ }^{3}$ I am very grateful to Philip Ryan for pointing out that $Y, Z$ provide the desired counterexample to the formula $j(h g)=h j(g)$.
} 
their action on connection forms in a way analogous to the action of their classical counterparts on the classical connection forms (see [4]). Quantum gauge transformations defined in this manner generalize locally defined quantum gauge transformations discussed in Section 3 of [9] (cf. Section 3 in [21]).

Definition 3.1 Let $P(B, A)$ be a quantum principal bundle. A k-homomorphism $f: A \rightarrow P$ is called a gauge transformation iff

1. $f$ is convolution invertible,

2. $\Delta_{R} \circ f=(f \otimes i d) \circ a d_{R}$,

3. $f(1)=1$.

Proposition 3.2 The set of all gauge transformations of a quantum principal bundle is a group with respect to convolution. We denote this group by $G T(P)$.

Proof. A routine sigma notation calculation verifies the following lemma:

Lemma 3.3 Let $\left\{\left(Q_{i}, \rho_{i}\right)\right\}_{i \in\{1,2\}}, \quad V_{12}$ and $m_{12}$ be as in Lemma 2.3. Then, for all $\mathrm{f}_{1} \in \operatorname{Hom}_{k}\left(A, Q_{1}\right), \mathrm{f}_{2} \in \operatorname{Hom}_{k}\left(A, Q_{2}\right)$,

$$
\left(\left(\mathrm{f}_{1} \otimes i d\right) \circ a d_{R}\right) *\left(\left(\mathrm{f}_{2} \otimes i d\right) \circ a d_{R}\right)=\left(\left(\mathrm{f}_{1} * \mathrm{f}_{2}\right) \otimes i d\right) \circ a d_{R} .
$$

Hence, since the map

$$
\left(\Delta_{R} \circ\right): \operatorname{Hom}_{k}(A, P) \ni \mathrm{f} \longmapsto \Delta_{R} \text { ○f } \in \operatorname{Hom}_{k}(A, P \otimes A)
$$

is an algebra homomorphism (cf. Lemma 4.0.2 in [35]), the set of all gauge transformations is closed under the convolution. Furthermore, it follows from the same reason that $\Delta_{R} \circ \mathrm{f}^{-1}=\left(\Delta_{R} \circ \mathrm{f}\right)^{-1}$. Therefore, as $f(1)=1$ implies $f^{-1}(1)=1$, by putting $\mathrm{f}_{1}=f$ and $\mathrm{f}_{2}=f^{-1}$ in Lemma 3.3, we can also conclude the existence of the inverse.

When defined in this way, quantum gauge transformations are unwilling to preserve the property $\omega\left(M_{A}\right)=0$ (see Definition 1.8 and Proposition 3.4) defining a connection form $\omega$ on a bundle with a general differential calculus. This is the case even if one assumes that the gauge transformations satisfy an additional condition $\left(\left(f \otimes f^{-1}\right) \circ \Delta\right)\left(M_{A}\right) \subseteq N_{P}$. (A related discussion can be found around formula (48) in 9]; note that this condition is satisfied in the classical situation, in which $M_{A}=(\operatorname{Ker} \varepsilon)^{2}$ — see Example 1 on p.132 in [39.) Therefore, when dealing with quantum gauge transformations, we will assume the universal differential calculus.

Proposition 3.4 Let $f \in G T(P)$ and $\omega \in \mathcal{C}(\mathcal{P})$, where $\mathcal{C}(\mathcal{P})$ denotes the space of all connection forms on a quantum principal bundle $\left(P, A, \Delta_{R}, 0,0\right)$. Then the formula

$$
G_{f} \omega=f * \omega * f^{-1}+f *\left(d \circ f^{-1}\right)
$$

(cf. (20) in 407) defines an action $G: G T(P) \times \mathcal{C}(\mathcal{P}) \longrightarrow \mathcal{C}(\mathcal{P})$. 
Proof. Let us verify first that $G_{f} \omega$ is indeed a connection form.

1. $G_{f} \omega(k)=0$. Obvious.

2. Taking into account that $\mathrm{T}$ is a left $P$-module morphism (see Point 4 in Definition 1.1) and remembering that for the universal differential calculus $N_{P}=0=M_{A}$, one can see that

$$
\begin{aligned}
& \left(\mathrm{T} \circ G_{f} \omega\right)(a) \\
& =f\left(a_{(1)}\right)(\mathrm{T} \circ \omega)\left(a_{(2)}\right)\left(\Delta_{R} \circ f^{-1}\right)\left(a_{(3)}\right) \\
& +\left((m \otimes i d) \circ\left(i d \otimes \Delta_{R}\right)\right)\left(f\left(a_{(1)}\right) \otimes f^{-1}\left(a_{(2)}\right)-\varepsilon(a) \otimes 1\right) \\
& =\left(f\left(a_{(1)}\right) \otimes\left(a_{(2)}-\varepsilon\left(a_{(2)}\right)\right)\right)\left(f^{-1}\left(a_{(4)}\right) \otimes S\left(a_{(3)}\right) a_{(5)}\right) \\
& +f\left(a_{(1)}\right) f^{-1}\left(a_{(3)}\right) \otimes S\left(a_{(2)}\right) a_{(4)}-1 \otimes \varepsilon(a) \\
& =f\left(a_{(1)}\right) f^{-1}\left(a_{(3)}\right) \otimes\left(\varepsilon\left(a_{(2)}\right) a_{(4)}-S\left(a_{(2)}\right) a_{(4)}\right) \\
& +f\left(a_{(1)}\right) f^{-1}\left(a_{(3)}\right) \otimes S\left(a_{(2)}\right) a_{(4)}-1 \otimes \varepsilon(a) \\
& =1 \otimes(a-\varepsilon(a)) .
\end{aligned}
$$

3. $\Delta_{\mathcal{R}} \circ G_{f} \omega=\left(G_{f} \omega \otimes i d\right) \circ a d_{R}$ can be proved much as Proposition 3.2.

Now, to complete the proof, it suffices to note that $G_{f * g} \omega=G_{f} G_{g} \omega$.

The action of the gauge group $G T(P)$ on the space of connections can be derived from its action on connection forms. It is explicitly described by

Proposition 3.5 Let $G T(P)$ be as in Proposition 3.4. Denote by $\mathcal{P}(P)$ the space of all connections on $P$, and by $\Upsilon$ the bijection providing the correspondence between connections and connection forms, i.e., let

$$
\Upsilon: \mathcal{P}(P) \ni \Pi \longmapsto \sigma_{\Pi} \circ(1 \otimes(i d-\varepsilon)) \in \mathcal{C}(\mathcal{P})
$$

where $\sigma_{\Pi}: P \otimes \operatorname{Ker} \varepsilon \rightarrow \Omega^{1} P$ is the unique left $P$-module homomorphism satisfying $\mathrm{\top} \circ \sigma_{\Pi}=i d$ and $\sigma_{\Pi} \circ \mathrm{T}=\Pi$. 囷 Then the map $\mathcal{G}: G T(P) \times \mathcal{P}(P) \longrightarrow \mathcal{P}(P)$ given by

$$
\mathcal{G}_{f} \Pi=m_{\Omega^{1} P} \circ\left(i d \otimes\left(\Pi \circ d \circ\left(i d *_{R} f\right)\right) *_{R} f^{-1}\right)+m_{\Omega^{1} P} \circ\left(i d \otimes f *\left(d \circ f^{-1}\right)\right) \circ \mathrm{\top}
$$

is the action of the gauge group $G T(P)$ on the space of connections $\mathcal{P}(P)$, and the following diagram commutes:

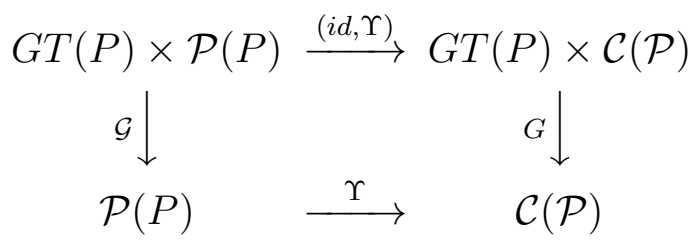

\footnotetext{
${ }^{4}$ See Proposition 4.4 and the paragraph above it in [9].
} 
Proof. Both assertions of the proposition follow from the formula:

$$
\forall f \in G T(P), \Pi \in \mathcal{P}(P): \mathcal{G}_{f} \Pi=\left(\Upsilon^{-1} \circ G_{f} \circ \Upsilon\right)(\Pi)
$$

Since both sides of the above equation are left $P$-module homomorphisms, in order to prove (28), it suffices to show that, for any $f \in G T(P)$ and $\Pi \in \mathcal{P}(\mathcal{P})$, we have

$$
\left(\mathcal{G}_{\{\diamond}\right)\left\lceil=\left(\left(\mp^{-\infty} \circ \mathcal{G}_{\{} \circ \mp\right)(\diamond)\right) \circ\lceil\right.
$$

that is,

$$
\begin{aligned}
& m_{\Omega^{1} P} \circ\left(i d \otimes f *\left(\sigma_{\Pi} \circ(1 \otimes(i d-\varepsilon))\right) * f^{-1}\right) \circ \mathrm{T} \circ d \\
& =m_{\Omega^{1} P} \circ\left(i d \otimes\left(\Pi \circ d \circ\left(i d *_{R} f\right)\right) *_{R} f^{-1}\right) \circ d
\end{aligned}
$$

(pure gauge terms cancel).

To do so, let us calculate the value of the left hand side of (29) on arbitrary $p \in P$ :

$$
\begin{aligned}
& \left(m_{\Omega^{1} P} \circ\left(i d \otimes f *\left(\sigma_{\Pi} \circ(1 \otimes(i d-\varepsilon))\right) * f^{-1}\right) \circ \mathrm{T} \circ d\right)(p) \\
& \left.=\left(m_{\Omega^{1} P} \circ\left(i d \otimes f *\left(\sigma_{\Pi} \circ(1 \otimes(i d-\varepsilon))\right) * f^{-1}\right)\right)\left(p_{(0)} \otimes p_{(1)}-p \otimes 1\right) \quad \text { (see }(24)[9]\right) \\
& =p_{(0)} f\left(p_{(1)}\right) \sigma_{\Pi}\left(1 \otimes p_{(2)}-\varepsilon\left(p_{(2)}\right) \otimes 1\right) f^{-1}\left(p_{(3)}\right) \\
& =\sigma_{\Pi}\left(p_{(0)} f\left(p_{(1)}\right) \otimes p_{(2)}-p_{(0)} f\left(p_{(1)}\right) \varepsilon\left(p_{(2)}\right) \otimes 1\right) f^{-1}\left(p_{(3)}\right) \\
& =\left(\sigma_{\Pi} \circ \top \circ d \circ\left(i d *_{R} f\right)\right)\left(p_{(0)}\right) f^{-1}\left(p_{(1)}\right) \\
& =\left(\left(\Pi \circ d \circ\left(i d *_{R} f\right)\right) *_{R} f^{-1}\right)(p) \\
& =\left(m_{\Omega^{1} P} \circ\left(i d \otimes\left(\Pi \circ d \circ\left(i d *_{R} f\right)\right) *_{R} f^{-1}\right)\right)(1 \otimes p-p \otimes 1) \\
& =\left(m_{\Omega^{1} P} \circ\left(i d \otimes\left(\Pi \circ d \circ\left(i d *_{R} f\right)\right) *_{R} f^{-1}\right) \circ d\right)(p)
\end{aligned}
$$

Hence, we can conclude that (29) is true, and the proposition follows.

Remark 3.6 The left $B$-module isomorphism $\left(i d *_{R} f\right): P \longrightarrow P$ can be regarded as a quantum version of a gauge transformation understood as an appropriate diffeomorphism of the total space of a principal bundle (see p.339 in [四] and Definition 5.1 in [7]). In fact, the map $f \mapsto i d *_{R} f$ is a group isomorphism (see Corollary 5.3 in [7]). (Observe that $\left(i d *_{R} f^{-1}\right) \circ\left(i d *_{R} f\right)=i d=$ $\left(i d *_{R} f\right) \circ\left(i d *_{R} f^{-1}\right)$. $)$

Having defined and described quantum gauge transformations and their action on the space of connections and connection forms, we can now show that these transformations preserve the strongness of a connection, i.e. (provided $M_{A}=0=N_{P}$ ) their action is well-defined on the space $\mathcal{S P}(P)$ of all strong connections on a quantum bundle $P$. (Obviously, their action is then also well-defined on the space $\mathcal{S C}(\mathcal{P})$ of all strong connection forms.)

Proposition 3.7 Let $P(B, A)$ be a quantum principal bundle with the universal differential calculus. Then $\forall f \in G T(P): \Pi \in \mathcal{S P}(P) \Leftrightarrow \mathcal{G}_{\{} \diamond \in \mathcal{S P}(P)$. 
Proof. Note first that, for any right $A$-comodule coaction $\rho_{\mathcal{C}}: \mathcal{C} \longrightarrow \mathcal{C} \otimes A$, any $\alpha_{1} \in \operatorname{Hom}_{k}\left(\mathcal{C}, \Omega^{m}(X)\right)$ and $\alpha_{2} \in \operatorname{Hom}_{k}\left(\mathcal{C}, \Omega^{n}(X)\right.$ ) (where $m \geq 0, n \geq 0$ and $\Omega(X)$ is any differential algebra), there is the familiar graded Leibniz rule:

$$
d \circ\left(\alpha_{1} *_{\rho_{\mathcal{C}}} \alpha_{2}\right)=\left(d \circ \alpha_{1}\right) *_{\rho_{\mathcal{C}}} \alpha_{2}+(-)^{m} \alpha_{1} *_{\rho_{\mathcal{C}}}\left(d \circ \alpha_{2}\right)
$$

Now, using similar calculations as in the proof of Proposition 3.5 and the Leibniz rule for the convolution, one can see that, for any $f \in G T(P), \Pi \in \mathcal{P}(P)$,

$$
\begin{aligned}
& \left(i d-\mathcal{G}_{f} \Pi\right) \circ d \\
& =d-\left(\Pi \circ d \circ\left(i d *_{R} f\right)\right) *_{R} f^{-1}-i d *_{R} f *\left(d \circ f^{-1}\right) \\
& =\left(d \circ\left(i d *_{R} f\right)\right) *_{R} f^{-1}-\left(\Pi \circ d \circ\left(i d *_{R} f\right)\right) *_{R} f^{-1} \\
& =\left((i d-\Pi) \circ d \circ\left(i d *_{R} f\right)\right) *_{R} f^{-1} .
\end{aligned}
$$

Hence, $\forall f \in G T(P): \Pi \in \mathcal{S P}(P) \Rightarrow \mathcal{G}_{f} \Pi \in \mathcal{S P}(P)$, and since $\mathcal{G}$ is a group action on $\mathcal{P}(P)$, $\forall f \in G T(P): \mathcal{G}_{f} \Pi \in \mathcal{S P}(P) \Rightarrow \Pi \in \mathcal{S P}(P)$.

\section{Curvature}

We are ready now to examine the properties of the exterior covariant derivative (see Definition 1.12) associated with a strong connection. This will lead to the definition of a (global) curvature form on a quantum principal bundle. The following proposition and corollaries describe the composition of the covariant exterior derivative with strongly tensorial differential forms (see Definition 1.11). They and Proposition 1.6 are analogous to the corresponding local (i.e. valid only for trivial quantum bundles) statements made in [9]. As we do not know how to characterize all differential algebras for which $D$ can be well-defined, we will simply assume here, whenever needed, that $\Omega(P)$ is a right-covariant differential algebra such that $D$ is well-defined.

Proposition 4.1 (cf. (17) and (76) in [9]) Let $\left(P, A, \Delta_{R}, N_{P}, M_{A}\right)$ be a quantum principal bundle with a connection form $\omega$, and let $\Omega(P)$ be a differential algebra such that $\Omega^{1}(P)=\Omega^{1} P / N_{P}$ and the exterior covariant derivative $D^{\omega}$ associated to $\omega$ is well-defined by formula (9). Then, for all $\varphi \in S T_{\rho}\left(V, \Omega^{n}(P)\right), n \in\{0\} \cup \mathbb{N}$,

$$
D^{\omega} \circ \varphi=d \circ \varphi-(-)^{n} \varphi *_{\rho} \omega .
$$

Proof. Note that, for any $d \alpha \in \Omega^{n}(B), n \in \mathbb{N}, p \in P$, we have

$$
\begin{aligned}
& \left(d-D^{\omega}\right)(d \alpha \cdot p) \\
& =(-)^{n} d \alpha d p-(-)^{n} d \alpha \cdot\left(i d-\Pi^{\omega}\right)(d p) \\
& =(-)^{n} d \alpha \cdot p_{(0)} \omega\left(p_{(1)}\right) \\
& =(-)^{n}\left(i d *_{\mathcal{R}} \omega\right)(d \alpha \cdot p) .
\end{aligned}
$$


On the other hand, for all $v \in V, \varphi(v)$ can be written as a finite sum $\sum_{i} d \alpha_{i} . p_{i}$ for some closed differential forms $d \alpha_{i} \in \Omega^{n}(B)$ and 0 -forms $p_{i} \in P$. Hence, with the help of Lemma 2.5, we obtain

$$
\left(\left(d-D^{\omega}\right) \circ \varphi\right)(v)=\left((-)^{n}\left(i d *_{\mathcal{R}} \omega\right) \circ \varphi\right)(v)=(-)^{n}\left(\varphi *_{\rho} \omega\right)(v),
$$

and the assertion follows.

Corollary 4.2 (cf. (7) 9 ) Let $\Omega(P)$ be as in the proposition above. If $D$ is the exterior covariant derivative associated to a strong connection, then, for every $n \in\{0\} \cup \mathbb{N}$, we have $D \circ\left(S T_{\rho}\left(V, \Omega^{n}(P)\right)\right) \subseteq S T_{\rho}\left(V, \Omega^{n+1}(P)\right)$.

Proof. In the same way as in the case of the differential envelope, it can be directly calculated that $D$ is always right-covariant (see Appendix A in [9]), and thus, for all connections, $D$ composed with a pseudotensorial differential form is a tensorial differential form, i.e.

$$
\forall n \in\{0\} \cup \mathbb{N}: D \circ\left(P T_{\rho}\left(V, \Omega^{n}(P)\right)\right) \subseteq T_{\rho}\left(V, \Omega^{n+1}(P)\right) .
$$

Hence, to prove the assertion of this corollary, it suffices to note that, if $D$ is associated to a strong connection, then, as can be seen from the second line of the first calculation in Proposition 4.1, $D$ evaluated on a strongly horizontal differential form yields a strongly horizontal differential form.

Remark 4.3 With the help of $D$ one can equivalently define a strong connection as a connection whose exterior covariant derivative maps $P$ into strongly horizontal forms, i.e. $D(P) \subseteq \Omega_{\text {shor }}^{1}(P)$. Obviously, since $D$ can be defined on $P$ for any differential calculus, such a definition works on any quantum principal bundle.

Corollary 4.4 (cf. Section 3 in [9]) Let $\omega$ be a strong connection form, and let $D^{\omega}$ and $\Omega(P)$ be as in Proposition 4.1 . Then

$$
\forall \varphi \in S T_{\rho}(V, \Omega(P)):\left(D^{\omega}\right)^{2} \circ \varphi=-\varphi *_{\rho}(d \circ \omega+\omega * \omega) .
$$

Formula (30) is, in particular, true for a classical principal bundle $\widetilde{P}(M, G)$. (A classical principal bundle is a special case of a quantum principal bundle when we replace the algebraic tensor product by the appropriately completed tensor product.) It is a generalization of the classical formula

$$
D^{2} \alpha=\varrho^{\prime}(F) \wedge \alpha
$$

where $\alpha$ is a differential form on $\widetilde{P}$ with values in a finite dimensional vector space $W$, the homomorphism $\varrho^{\prime}: \mathfrak{g} \rightarrow \mathfrak{g l}(\mathfrak{W})$ is the Lie algebra representation induced by a homomorphism $\varrho: G \rightarrow G L(W)$, and $F$ is the curvature form of a connection defining $D$ (e.g., see (19) in [37]). More precisely, taking $V$ to be the tensor algebra of the dual vector space $W^{*}$, defining $\rho_{R}$ by

$$
\forall v \in W^{*}: \rho_{R}(v)(w, g)=v\left(\varrho\left(g^{-1}\right) w\right),
$$

putting 


$$
\varphi_{\alpha}(v): \begin{cases}\bigwedge_{\tilde{p}}^{\operatorname{deg} \alpha} T_{\tilde{p}} \widetilde{P} X_{\tilde{p}} \longmapsto \begin{cases}(v \circ \alpha)\left(X_{\tilde{p}}\right) & \text { for } v \in W^{*} \\ 0 & \text { otherwise }\end{cases} & \text { for } \operatorname{deg} \alpha>0 \\ \widetilde{P} \ni \widetilde{p} \longmapsto(\check{v} \circ \alpha)(\widetilde{p}) \in \mathbb{R} & \text { for } \operatorname{deg} \alpha=0,\end{cases}
$$

where $\check{v}$ is the polynomial function on $W$ corresponding to the tensor $v$, and remembering that every $\mathfrak{g}$-valued differential form $\vartheta$ on $\widetilde{P}$ can be viewed as an $\varepsilon_{C^{\infty}(G)}$-derivation $\psi_{\vartheta}: C^{\infty}(G) \rightarrow \bigwedge^{*}(\widetilde{P})$ given by $\psi_{\vartheta}(a) X_{\tilde{p}}=\vartheta\left(X_{\tilde{p}}\right) a\left(\right.$ or $\psi_{\vartheta}(a) \widetilde{p}=\vartheta(\widetilde{p}) a$ if $\left.\operatorname{deg} \alpha=0\right)$, we can rewrite (31) as

$$
D^{2} \circ \varphi_{\alpha}=-\varphi_{\alpha} *_{\rho} F \text {. }
$$

Note that, since every vector space $W$ is a Lie group and there is a canonical isomorphism between $W$ and the Lie algebra of $W$, we could define $\varphi_{\alpha}$ in the same way as we define $\psi_{\vartheta}$. But then we would not have $\varphi_{\alpha}(1)=\delta_{0, \operatorname{deg} \alpha}$, which we need in Appendix A (see Proposition A.5). In the classical case, we can replace $F$ by $d \circ \omega+\omega * \omega$ or $D \circ \omega$ in the last formula, but we need to put $F=d \circ \omega+\omega * \omega$ to obtain (30). Also, formula (31) can be considered as a motivating factor in defining the curvature of a connection on a projective module as the square of a covariant derivative (see p.554 in [12]; covariant derivative $\equiv$ connection on a projective module). Therefore, it is natural to define the (global) curvature form of a connection $\Pi^{\omega}$ in the following way:

Definition 4.5 Let $\omega$ be a connection 1-form on a quantum principal bundle. The differential form dow $+\omega * \omega$ is called the curvature form of $\omega$ and is denoted by $F_{\omega}$.

Clearly, if $\omega$ is a strong connection form, then, at least in the case of a trivial bundle, for any differential algebra $\Omega(P)$, even if $D$ is not well-defined, $F_{\omega}$ is horizontal $\left(F_{\omega}=\Phi^{-1} * F_{\beta} * \Phi\right.$, where $\Phi$ and $\beta$ are as in Proposition 2.2 and $F_{\beta}:=d \circ \beta+\beta * \beta$ is a local curvature form, cf. (11) 9]). Moreover, unlike the expression $D \circ \omega$, the so-defined curvature form has the desired (at least from the point of view of Yang-Mills theory) transformation properties, i.e. we have:

Proposition 4.6 (cf. (13) [9], (20) [40]) Let $P(B, A)$ be a quantum principal bundle with the universal differential calculus, $\omega \in \mathcal{C}(\mathcal{P})$ and $f \in G T(P)$. Then

$$
F_{G_{f} \omega}=f * F_{\omega} * f^{-1} .
$$

Proof. Straightforward.

\section{$5 \quad U_{q}(2)$-Yang-Mills Theory on a Free Module}

To begin with, let us show that it is possible to define an action functional on quantum bundle connections in such a way that, at least in the case of a trivial quantum bundle, it agrees 
with the Yang-Mills action functional constructed in Section 1 of both [13] and [30]. (Clearly, we assume that the 'base space' of a quantum bundle is an algebra over which modules are considered in [13, 30].) Considerations in [8] and Proposition 4.6 suggest that, if $A$ is a matrix quantum group and $T$ its matrix of generators (fundamental representation, cf. p.628 in [38]), it is reasonable to define an action on a quantum principal bundle $P(B, A)$ to be (compare with the Lagrangian given by (6.65) in [19] or (4.1) in [21])

$$
Y M(\omega)=-\left(\mathcal{T} \circ \operatorname{Tr} \circ\left(F_{\omega} * F_{\omega}\right)\right)(T),
$$

where $\operatorname{Tr}$ is the usual matrix trace, and $\mathcal{T}: \Omega P \rightarrow k$ is a linear map vanishing on $[P, \Omega P]$. (To ensure that we have an ample supply of connections, throughout this section we will use the universal differential calculus.) Clearly, remembering the property of $T$ that, for any $\mathrm{f}_{1}$ and $\mathrm{f}_{2}$, $\left(\mathrm{f}_{1} * \mathrm{f}_{2}\right)\left(T_{a b}\right)=\sum_{c} \mathrm{f}_{1}\left(T_{a c}\right) \mathrm{f}_{2}\left(T_{c b}\right)$, one can see that

$$
\forall f \in G T(P), \omega \in \mathcal{C}(\mathcal{P}): \mathcal{Y} \mathcal{M}\left(\mathcal{G}_{\{} \omega\right)=\mathcal{Y} \mathcal{M}(\omega) .
$$

Similarly, if $P(B, A)$ is a trivial bundle with a trivialization $\Phi$ (which we will also assume for the rest of this section), then for any $\omega$,

$$
Y M(\omega)=-\left(\mathcal{T} \circ \operatorname{Tr} \circ\left(\Phi * F_{\omega} * F_{\omega} * \Phi^{-1}\right)\right)(T)=-\left(\mathcal{T} \circ \operatorname{Tr} \circ\left(F_{\beta} * F_{\beta}\right)\right)(T)=: Y M(\beta),
$$

where $\beta$ is given by formula (13) (see Remark 2.6) and $F_{\beta}$ is the curvature form associated to it (see the end of the previous section). As to $\mathcal{T}$, observe that one should expect to have a lot of information vested in it: projection from the universal to a non-universal calculus and metric (Hodge star). In what follows, we specify $\mathcal{T}$ in such a way as to incorporate the YangMills functional presented in [13, 30] into the quantum bundle framework. One ought to bear in mind that to obtain a $q$-deformed Yang-Mills theory in the spirit of quantum groups, one should invent another $\mathcal{T}$ or change the formula (32) altogether. Here, however, we investigate what effects can entail from the deformation of the structure group alone.

Definition 5.1 Let $\mathcal{T}_{B}$ be a faithful invariant trace on $B$ (as in [13, 30]), $\mathcal{L}$ be a finite dimensional Lie subalgebra of $\operatorname{Der}(B), \quad\left\{X_{l}\right\}_{l \in\{1, \ldots, \operatorname{dim} \mathcal{L}\}}$ be its basis, and $\widetilde{X}_{l}=X_{l} \otimes i d$, $l \in\{1, \ldots, \operatorname{dim} \mathcal{L}\}$. We put

$$
\mathcal{T}(\alpha)= \begin{cases}\left(\mathcal{T}_{B} \otimes \varepsilon\right)(\alpha) & \text { if } \operatorname{deg} \alpha=0 \\ 0 & \text { if } \operatorname{deg} \alpha=2 m-1 \\ \sum_{l_{1}<\cdots<l_{m}}\left(\left(\mathcal{T}_{B} \otimes \varepsilon\right) \circ \alpha\right)\left(\widetilde{X}_{l_{1}} \wedge \cdots \wedge \widetilde{X}_{l_{m}} \otimes \widetilde{X}_{l_{1}} \wedge \cdots \wedge \widetilde{X}_{l_{m}}\right) & \text { if } \operatorname{deg} \alpha=2 m,\end{cases}
$$

where $m \in \mathbb{N}$.

Recall that the product of differential 1-forms evaluated on the tensor product of derivations is, as in the classical case, given by

$$
\left(\alpha_{1} \cdots \alpha_{n}\right)\left(Y_{1} \otimes \cdots \otimes Y_{n}\right)=\alpha_{1}\left(Y_{1}\right) \cdots \alpha_{n}\left(Y_{n}\right)
$$

and $\left(b_{0} d b_{1}\right)(Y)=b_{0} Y\left(b_{1}\right)$ (see p.403 in [16]). (Observe that, as $\left\{Y_{i}\right\}_{i \in\{1, \cdots, n\}}$ are derivations, it does not matter in which way we write a differential form as a sum of products of differential 1-forms.) Now, with $\mathcal{T}$ defined as above, we have $\mathcal{T}([P, \Omega P])=0$. Moreover, we have 
Proposition 5.2 Let $A$ and $T$ be as above. Also, let $B$ be a *-algebra, $P(B, A)$ a trivial quantum principal bundle with a trivialization $\Phi$ (e.g., $P=B \otimes A$ ), $\omega$ a strong connection form on $P$, and $\mathcal{T}$ as in Definition 5.1. Then

$$
Y M(\omega)=Y M\left(\nabla^{\omega}\right)
$$

where $\nabla^{\omega}=d+\beta(T)$ is the $\omega$ induced connection on the right module $B^{n}$ ( $\beta$ is given by (19), $n$ is the size of $T, \nabla^{\omega} \xi=d \xi+\beta(T) \xi$ - see p.637 in (97), and the right hand side of (34) is defined in Definition 1.20.

Proof. Note first that, by Proposition 2.2, $\beta(T) \in M_{n}\left(\Omega^{1} B\right)$. Consequently, $F_{\beta}(T)=$ $d \beta(T)+\beta(T)^{2}$ is an element of $M_{n}\left(\Omega^{2} B\right)$ (see the paragraph above Proposition 4.6 for $F_{\beta}$ ). Therefore, since the curvature $\left(\nabla^{\omega}\right)^{2}$ equals just $d \beta(T)+\beta(T)^{2}$ (cf. p.681 in [11]), with the help of (33), Corollary 1.19 and Proposition 1.13, we have

$$
\begin{aligned}
Y M(\omega) & =Y M(\beta) \\
& =-\sum_{r<s}\left(\left(\mathcal{T}_{\mathcal{B}} \otimes \varepsilon\right) \circ \mathcal{T} \nabla\left(\mathcal{F}_{\beta} * \mathcal{F}_{\beta}\right)(\mathcal{T})\right)\left(\widetilde{\mathcal{X}}_{\nabla} \wedge \widetilde{\mathcal{X}}_{f} \otimes \widetilde{\mathcal{X}}_{\nabla} \wedge \widetilde{\mathcal{X}}_{f}\right) \\
& =-\sum_{\substack{i, j \\
r<s}}\left(\mathcal{T}_{B} \circ\left(F_{\beta}\left(T_{i j}\right) F_{\beta}\left(T_{j i}\right)\right)\right)\left(X_{r} \wedge X_{s} \otimes X_{r} \wedge X_{s}\right) \\
& =-\left(\mathcal{T}_{B} \circ T r\right)\left(\sum_{r<s} F_{\beta}(T)\left(X_{r} \wedge X_{s}\right) F_{\beta}(T)\left(X_{r} \wedge X_{s}\right)\right) \\
& =-\mathcal{T}_{\mathcal{E}}\left(\sum_{\nabla<j}\left(\left(\nabla^{\omega}\right)^{\epsilon}\left(\mathcal{X}_{\nabla} \wedge \mathcal{X}_{f}\right)\right)^{\epsilon}\right) \\
& =-\mathcal{T}_{\mathcal{E}}\left\{\times_{\nabla^{\omega}}, \times_{\nabla^{\omega}}\right\}=\mathcal{Y M}\left(\nabla^{\omega}\right) .
\end{aligned}
$$

Thus, the Yang-Mills action functional given by (32) coincides, as stated above, with the YangMills action functional defined in [13, 30] (cf. Section VII.D in [17] and Section V.B in [18]). This confirms that, as was mentioned in [9], the formalism of quantum group gauge theory is "not incompatible with the existing ideas in non-commutative geometry".

Remark 5.3 Observe that although any trivial quantum bundle $P(B, A)$ is isomorphic with $B \otimes A$ (algebras $B$ and $B \otimes 1$ identified) as a left $B$-module, we cannot claim in general that it is isomorphic with $B \otimes A$ as an algebra (see the first paragraph of Section 3.1 in [28]). But here, since our attention is restricted to strong connections, the structure of the 'total space' is not important - we can equally well define the Yang-Mills action functional by

$$
Y M(\beta)=-\left(\mathcal{T}_{\Omega B} \circ \operatorname{Tr} \circ\left(F_{\beta} * F_{\beta}\right)\right)(T),
$$

where $\mathcal{T}_{\Omega B}$ is a $k$-linear map vanishing on $[B, \Omega B]$. (Obviously, the Yang-Mills action thus defined is invariant under the local gauge transformations, i.e. gauge transformations taking values in $B$ rather than in $P$; see Definition 3.1). 
Remark 5.4 Note that the derivations used in Definition 5.1 are not fully antisymmetrized there is a tensor product in between two groups of antisymmetrized derivations. This is a key difference between the construction of $Y M(\nabla)$ and the construction of the second Chern class. (One should think of $\mathcal{T}$ defined in Definition 5.1 as a pairing rather than a trace.)

Now, we are going to take a closer look at what happens if we choose $A=U_{q}(2)$ (with $q \in \mathbb{R}_{+}$). (We continue to assume, as in Proposition 5.2, that B is a $*$-algebra, so that $\Omega(B)$ is also a *-algebra; cf. (1.32) in [39.) To do so, let us first recall the definition of $U_{q}(2)$ (see Lecture 5 in [36]):

Definition $5.5\left(U_{q}(2), \Delta, \varepsilon, S\right)$ is a matrix $*$-Hopf algebra determined by the following equalities:

$$
U_{q}(2)=\mathbb{C}\left\langle a, b, t, a^{*}, b^{*}, t^{*}, 1\right\rangle /\left(\mathcal{J}+\mathcal{J}^{*}\right),
$$

where

$$
\begin{aligned}
\mathcal{J}= & \left(a b-q b a, b b^{*}-b^{*} b, a b^{*}-q b^{*} a, a^{*} a-a a^{*}+\left(q^{-2}-1\right) b b^{*},\right. \\
& \left.t a-a t, t b-b t, t a^{*}-a^{*} t, t b^{*}-b^{*} t, t t^{*}-t^{*} t, a a^{*}+b b^{*}-1, t t^{*}-1\right)
\end{aligned}
$$

and $q \in \mathbb{R} \backslash\{0\} ; 5$

$$
\Delta T=T \dot{\otimes} T, \quad \varepsilon(T)=I, \quad S(T)=T^{\dagger},
$$

where

$$
T=\left(\begin{array}{cc}
a & b \\
-q^{-1} b^{*} t^{*} & a^{*} t^{*}
\end{array}\right)
$$

and $\dot{\otimes}$ is the matrix multiplication with the product of its entries replaced by the tensor product $\otimes$; and

$$
\Delta t=t \otimes t, \quad \varepsilon(t)=1, \quad S(t)=t^{*}, \quad S\left(t^{*}\right)=t .
$$

The next step is to establish an equivalence between a certain class of strong connections on a trivial $U_{q}(2)$-bundle and the space of hermitian connections on the associated free module. Since any $\beta \in \operatorname{Hom}_{\mathbb{C}}\left(U_{q}(2), \Omega B\right)$ satisfying $\beta(\mathbb{C})=0$ is a connection form on the base space $B$ of a trivial $U_{q}(2)$ bundle (see Remark 2.6 and the paragraph below) and the $\beta$-induced connection $\nabla$ on $B^{2}$ depends only on $\beta(T)$, we will restrict our attention to connection forms that are, in some way, uniquely determined by $\beta(T)$. In the classical situation, $\beta$ is an $\varepsilon$-derivation and thus is automatically determined by $\beta(T)$. Mimicking this classical differential geometry formula for $\beta$, we define an auxiliary map $\widetilde{\beta}: \mathbb{C}\left\langle\partial,, \approx, \partial^{*},{ }^{*}, \approx^{*}, \nVdash\right\rangle \rightarrow \Omega^{\nVdash} \mathbb{B}$ by

$$
\widetilde{\beta}\left(a^{k_{1}} a^{* l_{1}} b^{m_{1}} b^{* n_{1}} t^{p_{1}} t^{* r_{1}} \cdots a^{k_{s}} a^{* l_{s}} b^{m_{s}} b^{* n_{s}} t^{p_{s}} t^{* r_{s}}\right)
$$

\footnotetext{
5 Since, further on, we want $\left(\begin{array}{ll}1 & 0 \\ 0 & q\end{array}\right)$ to be positive definite, we actually need to assume that $q>0$.
} 


$$
= \begin{cases}0 & \text { for } \sum_{i=1}^{s} m_{i}+n_{i} \geq 2 \\ q^{\sum_{i=j+1}^{s}\left(l_{i}-k_{i}\right)} \widetilde{b} & \text { for } m_{j}=1, j \in\{1, \ldots, s\}, \sum_{i=1}^{s} m_{i}+n_{i}=0 \\ q^{\sum_{i=j+1}^{s}\left(l_{i}-k_{i}\right)} \widetilde{b}^{*} & \text { for } n_{j}=1, j \in\{1, \ldots, s\}, \sum_{i=1}^{s} m_{i}+n_{i}=0 \\ \sum_{i=1}^{s} k_{i} \widetilde{a}+\sum_{i=1}^{s} l_{i} \widetilde{a}^{*}+\sum_{i=1}^{s} p_{i} \widetilde{t}+\sum_{i=1}^{s} r_{i} \widetilde{t}^{*} & \text { for } \sum_{i=1}^{s} m_{i}+n_{i}=0,\end{cases}
$$

where, a priori, $\widetilde{a}, \widetilde{b}$ and $\widetilde{t}$ are any differential forms in $\Omega^{1} B$. One can verify that $\widetilde{\beta}\left(\mathcal{J}+\mathcal{J}^{*}\right)=$, if $\widetilde{a}+\widetilde{a}^{*}=0=\widetilde{t}+\widetilde{t}^{*}$. Hence, since

$$
\widetilde{\beta}\left(a a^{*}+b b^{*}-1\right)=0=\widetilde{\beta}\left(t t^{*}-1\right) \Rightarrow \widetilde{a}+\widetilde{a}^{*}=0=\widetilde{t}+\widetilde{t}^{*},
$$

we can conclude that (35) defines $\beta \in \operatorname{Hom}_{\mathbb{C}}\left(U_{q}(2), \Omega^{1} B\right)$ such that $\beta(1)=0$ (i.e., a $U_{q}(2)$-connection form) if and only if $\widetilde{a}+\widetilde{a}^{*}=0=\widetilde{t}+\widetilde{t}^{*}$. Moreover, it is straightforward to check that

$$
\beta(T)^{\dagger}\left(\begin{array}{ll}
1 & 0 \\
0 & q
\end{array}\right)+\left(\begin{array}{ll}
1 & 0 \\
0 & q
\end{array}\right) \beta(T)=0,
$$

and conversely, that for every $M \in M_{2}\left(\Omega^{1} B\right)$ satisfying (36) we can find unique $\widetilde{a}, \widetilde{b}, \widetilde{t}$ such that $\beta(T)=M$ (cf. Proposition 1 in [3]). Thus we have proved the following:

Proposition 5.6 Let $\mathcal{C}_{\mathcal{B}}=\left\{\beta \in \operatorname{Hom}_{\mathbb{C}}\left(\mathcal{U}_{\mathrm{II}}(\in), \Omega^{\infty} \mathcal{B}\right) \mid \beta\right.$ satisfies (3ף) for some $\left.\widetilde{\jmath}, \widetilde{L}, \widetilde{\sqcup} \in \Omega^{\infty} \mathcal{B}\right\}$ and $\mathcal{C C}\left(\mathcal{B}^{\epsilon}\right)$ denote the space of Hermitian connections on $B^{2}$ (see Point 1 and Point 3 of Definition 1.15), where

$$
\beta\left(a^{k} a^{* l} b^{m} b^{* n} t^{p} t^{* r}\right):= \begin{cases}0 & \text { for } m+n \geq 2 \\ \widetilde{b} & \text { for } m=1, n=0 \\ \widetilde{b}^{*} & \text { for } m=0, n=1 \\ (k-l) \widetilde{a}+(p-r) \widetilde{t} & \text { for } m=n=0,\end{cases}
$$

and $\langle$,$\rangle is given by the formula \langle\xi, \zeta\rangle=\xi^{\dagger}\left(\begin{array}{ll}1 & 0 \\ 0 & q\end{array}\right) \zeta$. The map

$$
\psi: \mathcal{C}_{\mathcal{B}} \ni \beta \longmapsto \Gamma+\beta(\mathcal{T}) \in \mathcal{C C}\left(\mathcal{B}^{€}\right)
$$

is a bijection.

Corollary 5.7 Let $P\left(B, U_{q}(2)\right)$ be a trivial quantum principal bundle with the universal differential calculus. There exists a one-to-one correspondence between the elements of $\mathcal{C C}\left(\mathcal{B}^{\epsilon}\right)$, i.e. the universal calculus connections on $B^{2}$ that are compatible with the Hermitian metric given by the matrix $\left(\begin{array}{ll}1 & 0 \\ 0 & q\end{array}\right)$, and the strong connections on $P\left(B, U_{q}(2)\right)$ whose 'pullback' on the base space $B$ (given by (13)) satisfies (37). 
This way, we obtain the Yang-Mills theory of connections compatible with the $q$-dependent Hermitian structure on $B^{2}$. Now, in order to handle the critical points of $Y M$ the same way they were dealt with in [30], let us assume (as in [30], p.535-6) that $B$ is a smooth dense $*$-subalgebra of a $C^{*}$-algebra, and that it is equipped with a faithful invariant trace $\mathcal{T}_{B}$. As was argued in Section 1 of [13], the Yang-Mills functional does not depend on the choice of a Hermitian metric - we can gauge $q$ out of the picture. Hence, the critical points of the $U_{q}(2)$-Yang-Mills action functional are simply the critical points of the Yang-Mills action (see p.536 in [30]), if there are any, 'rotated' by the appropriate $q$-dependent gauge transformation (see Section 1 in [13). (More explicitly, as the Hermitian metric is given by $\left(\begin{array}{ll}1 & 0 \\ 0 & q\end{array}\right)$, the corresponding gauge transformation is $\nabla \mapsto\left(\begin{array}{cc}1 & 0 \\ 0 & \sqrt{q}\end{array}\right)^{-1} \nabla\left(\begin{array}{cc}1 & 0 \\ 0 & \sqrt{q}\end{array}\right)$.) Consequently, we have:

Corollary 5.8 In the above described setting, the $U_{q}(2)$ and $U(2)-Y a n g-M i l l s$ theories have the same moduli spaces of critical points (cf. Section 4 in [30] and p.582 in [19]).

Another way to remove $q$ from the picture is to alter (37) by replacing $\widetilde{b}^{*}$ by $q \widetilde{b}^{*}$. Then, however, we would lose the geometrical interpretation of the action of the $q$-deformation of $U(2)$ on the space of compatible connections as the action of the gauge transformation. Also, the formula $\beta\left(T^{*}\right)=\beta(T)^{*}$ would no longer be true. (Caution: at least in the general case, we cannot claim that $\beta$ is a $*$-morphism even when it commutes with the $*$ on the generators.) In any case, we can see that the Yang-Mills theory remains unchanged for any $q \in \mathbb{R}_{+}$. A similar situation was discussed in the context of quantum group gauge theories on classical spaces in the last two paragraphs of Section 2 in [8].

Remark 5.9 The reason for employing in the considerations above $U_{q}(2)$ rather than $S U_{q}(2)$ is that, when using $S U_{q}(2)$, formula (37) entails $\operatorname{Tr} \beta(T)=0$ for all $\beta$, and although the tracelessness of $\beta(T)$ is automatically preserved by the entire $G L\left(B^{2}\right)$ in the classical case, we cannot claim the same in general (cf. Introduction and Section 3 in [2]). Nor can we claim that the tracelessness of $\beta(T)$ is preserved by the gauge group $U\left(B^{2}\right)$ of unitary automorphisms of $B^{2}$ (see Remark 1.16). Besides, $U_{q}(2)$-connections given by (37) can be regarded as $\langle$,$\rangle -compatible$ connections, and vice versa, which makes a clear analogy with the classical situation, where Hermitian metrics are $U(2)$-structures (cf. p.13 in [37]), and $U(2)$-connections are automatically compatible with the corresponding Hermitian structures (cf. p.94-5 in [37).

Finally, let us mention that an alternative approach would be to work with a non-universal differential calculus instead of assuming (35), which is put in the theory by hand. But that is yet another story.

\section{Appendix}

\section{A Quantum Associated Bundles}


Definition A.1 (cf. A.3 [9]) Let $\left(P, A, \Delta_{R}, N_{P}, M_{A}\right)$ be a quantum principal bundle and $\left(V, \rho_{R}\right)$ be a right $A^{o p}$-comodule algebra (see the remark below). Also, let $\Delta_{E}$ be the homomorphism from $P \otimes V$ into $P \otimes V \otimes A$ given by

$$
\Delta_{E}=(i d \otimes i d \otimes(m \circ \tau)) \circ(i d \otimes \tau \otimes i d) \circ\left(\Delta_{R} \otimes \rho_{R}\right),
$$

and $E$ be the space of all right-invariant elements of $P \otimes V$, i.e.

$$
E=\left\{t \in P \otimes V \mid \Delta_{E} t=t \otimes 1\right\}
$$

Then $\left(E, P, V, \rho_{R}\right)$ is called the quantum fiber bundle associated by $\rho_{R}$ to the quantum principal bundle $P$.

Remark A.2 Note that the notion of a comodule algebra makes sense for any bialgebra. In the preceding definition, we do not assume that $A^{o p}$ is a Hopf algebra. Such an assumption is equivalent to an assumption that the antipode of $A$ is bijective - a restriction that is unnecessary here. (Contrary to [9], we use $S$ rather than $S^{-1}$.) Observe also that the homomorphism $\Delta_{E}$ makes $P \otimes V$ a right $A$-comodule.

We call $E$ a quantum fiber bundle instead of a quantum vector bundle, as it is called in [9]. The only difference, however, between Definition A.3 [9] and Definition A.1 is an extra twist in the formula defining $\Delta_{E}$ (i.e. $m$ is replaced by $m \circ \tau$; for comparison see also Corollary 4.14 in 28 and Section 5 in [10]). An advantage of introducing such a change is that, from the point of view of further constructions, it is consistent with defining strongly horizontal differential forms as elements of $\Omega(B) P$ rather than $P \Omega(B)$. As to using the word "fiber" rather than "vector", the reason for doing so is that, since in order to define section-valued differential forms $\Gamma^{*}(E)$ (Definition A.4) or to prove the propositions presented here it is unnecessary to assume that $V$ is a quantum vector space (quadratic algebra; see [27]), we formulate everything without this assumption, and treat $V$ as a 'quantum fiber'. (See the paragraph below Corollary 4.4 for how, having a linear structure on a fiber, one can reproduce the familiar classical situation of vector valued differential forms.) The following are the reformulations of the corresponding statements in [9]. Except for the last parts of the proofs of Proposition A.5 and Proposition A.8, the remaining proofs in this section are straightforward modifications of the corresponding proofs in $[9]$.

Proposition A.3 (cf. Lemma A.4 [9]) The spaces $E$ and $B \otimes 1$ (see Definition 1.1) are subalgebras of $P \otimes V$ and $E$ respectively.

Proof. Analogous to the proof of Lemma A.4[9].

Many statements below concern differential forms of an arbitrary degree. Therefore, we need to assume that we have a quantum principal bundle $P(B, A)$ equipped with a differential algebra $\Omega(P)$ such that $\Omega^{1}(P)$ is the first order differential calculus of $P(B, A)$.

\footnotetext{
${ }^{6}$ I owe noticing this point to Marc Rieffel.
} 
Definition A.4 (cf. (69)[9]) A k-linear map $s: E \rightarrow \Omega^{n}(B)$, with $n \in\{0\} \cup \mathbb{N}$, satisfying $\forall b \in B: s \circ \widetilde{R}_{b \otimes 1}=R_{b} \circ s$ and $s(1 \otimes 1)=\delta_{n, 0}$, where $\widetilde{R}$ and $R$ denote the corresponding multiplications on the right, is called a differential form with values in sections of the quantum fiber bundle $E$. (For $n=0, s$ is simply called a section of a quantum fiber bundle.) The space of all such $n$-forms will be denoted by $\Gamma^{n}(E)$.

Proposition A.5 (cf. Proposition A.5 [9]) Let $\varphi \in S T_{\rho}\left(V, \Omega^{n}(P)\right), n \in\{0\} \cup \mathbb{N}$, be such that $\varphi(1)=\delta_{\operatorname{deg} \varphi, 0}$ (the space of all such forms will be denoted by $\overline{S T}_{\rho}\left(V, \Omega^{n}(P)\right)$ ) and let $s:=\left.m_{\Omega(P)} \circ \tau \circ(i d \otimes \varphi)\right|_{E}$. Then, if $\operatorname{deg} \varphi=0$ or if $P(B, A)$ is a trivial bundle, $s \in \Gamma^{\operatorname{deg} \varphi}(E)$.

Proof. To begin with, observe that for any $\varphi \in \overline{S T}_{\rho}\left(V, \Omega^{n}(P)\right), n \in\{0\} \cup \mathbb{N}$ and $b \in B$, we have $s \circ \widetilde{R}_{b \otimes 1}=R_{b} \circ s$ and $s(1 \otimes 1)=\delta_{\operatorname{deg} s, 0}$, so that it only remains to be shown that $s(E) \subseteq \Omega^{\operatorname{deg} \varphi}(B)$. Note also that, if $\sum_{i} p^{i} \otimes v^{i} \in E$, we have

$$
\left(\Delta_{\mathcal{R}} \circ m_{\Omega(P)} \circ \tau \circ(i d \otimes \varphi)\right)\left(\sum_{i} p^{i} \otimes v^{i}\right)=\left(\left(m_{\Omega(P)} \circ \tau \circ(i d \otimes \varphi)\right)\left(\sum_{i} p^{i} \otimes v^{i}\right)\right) \otimes 1 .
$$

(We write the sums because we cannot claim that $E$ is spanned by simple tensors.) Now, since it is clear that $s(E) \subseteq \Omega_{\text {shor }}^{\operatorname{deg} \varphi}(P)$, we can conclude that $s$ takes values in strongly horizontal right-invariant differential forms on $P$. Hence, if $\operatorname{deg} \varphi=0$, then $s(E) \subseteq B$, and it follows that $s \in \Gamma^{0}(E)$. On the other hand, if there exists a trivialization of a quantum principal bundle $P(B, A)$, by the same reasoning as was used to justify formula (18), we can infer that strongly horizontal right-invariant differential forms must be elements of $\Omega(B)$. Consequently, $s \in \Gamma^{\operatorname{deg} \varphi}(E)$ as claimed. (Warning: As of now, we do not know whether or not it is always true that every right-invariant strongly horizontal form on $P$ is a form on $B$. Hence, as a precaution, we assume the triviality of $P(B, A)$ when dealing with forms of degree bigger than 0.)

For the remaining two propositions we will assume that $P(B, A)$ is a trivial quantum principal bundle with a trivialization $\Phi$.

Proposition A.6 (cf. (71) and (72) in [9]) Let $\Phi_{E}:=((\Phi \circ S) \otimes i d) \circ \tau \circ \rho_{R}$. Then

$$
\mathcal{I}:=\left(m_{P} \otimes i d\right) \circ\left(i d \otimes \Phi_{E}\right)
$$

is a left $P$-module automorphism of $P \otimes V$. Its inverse is given by

$$
\widetilde{\mathcal{I}}:=\left(m_{P} \otimes i d\right) \circ\left(i d \otimes\left(\Phi^{-1} \circ S\right) \otimes i d\right) \circ\left(i d \otimes\left(\tau \circ \rho_{R}\right)\right) .
$$

Moreover, $\Phi_{E}(V) \subseteq E$ and $\widetilde{\mathcal{I}}(E)=B \otimes V$.

\section{Proof.}

1. It is straightforward to check that $\widetilde{\mathcal{I}} \circ \mathcal{I}=i d=\mathcal{I} \circ \widetilde{\mathcal{I}}$. Since $\mathcal{I}$ is, clearly, a left $P$-module homomorphism, we can now conclude that $\mathcal{I}$ is a left $P$-module automorphism of $P \otimes V$.

2. A direct sigma notation calculation shows that $\Delta_{E} \circ \Phi_{E}=\Phi_{E} \otimes 1$, whence $\Phi_{E}(V) \subseteq E$.

3. Finally, we must show that $\widetilde{\mathcal{I}}(E)=B \otimes V$. To do so, first we need: 
Lemma A.7 (cf. 3.1 31] and (72) [9]) For every $\sum_{i} p^{i} \otimes v^{i} \in E$,

$$
\sum_{i} p_{(0)}^{i} \otimes v^{i} \otimes p_{(1)}^{i}=\sum_{i} p^{i} \otimes v_{(0)}^{i} \otimes S\left(v_{(1)}^{i}\right)
$$

Proof. This lemma can be verified by applying

$$
(i d \otimes i d \otimes m) \circ(i d \otimes i d \otimes S \otimes i d) \circ\left(i d \otimes \rho_{R} \otimes i d\right)
$$

to both sides of the equality $\sum_{i} p_{(0)}^{i} \otimes v_{(0)}^{i} \otimes v_{(1)}^{i} p_{(1)}^{i}=\sum_{i} p^{i} \otimes v^{i} \otimes 1$.

Now, with the help of the above lemma, one can see that $\left.\widetilde{\mathcal{I}}\right|_{E}=\mathrm{s}_{\Phi} \otimes i d$ (see (20)), whence $\widetilde{\mathcal{I}}(E) \subseteq B \otimes V$. Furthermore, as $B \otimes 1$ is a subalgebra of $E$ (see Proposition A.3), and $\Phi_{E}(V) \subseteq E$, we also have $\mathcal{I}(B \otimes V) \subseteq E$. Thus, since $\widetilde{\mathcal{I}} \circ \mathcal{I}=i d$, it follows that $\widetilde{\mathcal{I}}(E)=B \otimes V$.

Closing our list of propositions is:

Proposition A.8 Let $\widetilde{\Phi}_{E}:=\left(\Phi^{-1} \otimes i d\right) \circ \tau \circ \rho_{R}\left(c f .\left(\right.\right.$ (73)[G]). Then $\widetilde{\Phi}_{E}(V) \subseteq E$, and

$$
\Psi: \Gamma^{*}(E) \ni s \longmapsto\left(s \circ \widetilde{\Phi}_{E}\right) *_{\rho} \Phi \in \overline{S T}_{\rho}\left(V, \Omega^{*}(P)\right)
$$

is a bijection. П Its inverse is given by

$$
\widetilde{\Psi}:\left.\overline{S T}_{\rho}\left(V, \Omega^{*}(P)\right) \ni \varphi \longmapsto m_{\Omega(P)} \circ \tau \circ(i d \otimes \varphi)\right|_{E} \in \Gamma^{*}(E) .
$$

\section{Proof.}

1. Using formula (28) 9] (see the proof of Corollary 2.4), one can prove the inclusion $\widetilde{\Phi}_{E}(V) \subseteq E$ in the same way as the inclusion $\Phi_{E}(V) \subseteq E$, i.e. by a direct sigma notation calculation. (Observe that $\widetilde{\Phi}_{E}$ and $\Phi_{E}$ coincide in the classical case.)

2. We already know from Proposition A.5 that $\widetilde{\Psi}$ indeed takes values in $\Gamma^{*}(E)$. Furthermore, it is clear that

$$
\forall s \in \Gamma^{n}(E), n \in\{0\} \cup \mathbb{N}: \Psi(s)(V) \subseteq \Omega_{\text {shor }}^{n}(P) \text { and } \Psi(s)(1)=\delta_{n, 0} .
$$

Proceeding much as in the first calculation in the proof of Proposition A.7 [9], we can show that, for an arbitrary $s \in \Gamma^{n}(E), n \in\{0\} \cup \mathbb{N}$, we have

$$
\Delta_{\mathcal{R}} \circ \Psi(s)=(\Psi(s) \otimes i d) \circ \rho_{R} .
$$

Hence the inclusion $\Psi\left(\Gamma^{*}(E)\right) \subseteq \overline{S T}_{\rho}\left(V, \Omega^{*}(P)\right)$ follows.

\footnotetext{
7 This isomorphism corresponds to the formula for a pseudotensorial 0 -form on $P$ that one could obtain by combining the very last formula in the proof of Corollary A.8 and formula (74) in [9].
} 
3. For an arbitrary $s \in \Gamma^{n}(E), n \in\{0\} \cup \mathbb{N}$ and $\sum_{i} p^{i} \otimes v^{i} \in E$, we have

$$
\begin{aligned}
& (\widetilde{\Psi} \circ \Psi)(s)\left(\sum_{i} p^{i} \otimes v^{i}\right) \\
& =\sum_{i}\left(m_{\Omega(P)} \circ \tau \circ\left(i d \otimes\left(\left(s \circ \widetilde{\Phi}_{E}\right) *_{\rho} \Phi\right)\right)\right)\left(p^{i} \otimes v^{i}\right) \\
& =\sum_{i}\left(s \circ \widetilde{\Phi}_{E}\right)\left(v_{(0)}^{i}\right) \Phi\left(v_{(1)}^{i}\right) p^{i} \\
& =\sum_{i}\left(i d *_{\mathcal{R}} \Phi^{-1}\right)\left(\left(s \circ \widetilde{\Phi}_{E}\right)\left(v_{(0)}^{i}\right) \Phi\left(v_{(1)}^{i}\right) p^{i}\right) \quad(\operatorname{Im}(\widetilde{\Psi} \circ \Psi)(s) \subseteq \Omega(B)) \\
& =\sum_{i}\left(s \circ \widetilde{\Phi}_{E}\right)\left(v_{(0)}^{i}\right) \mathrm{S}_{\Phi}\left(\Phi\left(v_{(1)}^{i}\right) p^{i}\right) \\
& \begin{array}{l}
=\sum_{i} s\left(\widetilde{\Phi}_{E}\left(v_{(0)}^{i}\right)\left(\mathrm{s}_{\Phi}\left(\Phi\left(v_{(1)}^{i}\right) p^{i}\right) \otimes 1\right)\right) \\
=\sum_{i} s\left(\left(\Phi^{-1}\left(v_{(1)}^{i}\right) \otimes v_{(0)}^{i}\right)\left(\Phi\left(v_{(2)}^{i}\right) p_{(0)}^{i} \Phi^{-1}\left(v_{(3)}^{i} p_{(1)}^{i}\right) \otimes 1\right)\right)
\end{array} \\
& =\sum_{i} s\left(p_{(0)}^{i} \Phi^{-1}\left(v_{(1)}^{i} p_{(1)}^{i}\right) \otimes v_{(0)}^{i}\right) \\
& =(s \circ \mathrm{F})\left(\sum_{i} p_{(0)}^{i} \otimes v^{i} \otimes p_{(1)}^{i}\right) \\
& =(s \circ \mathrm{F})\left(\sum_{i} p^{i} \otimes v_{(0)}^{i} \otimes S\left(v_{(1)}^{i}\right)\right) \\
& \left.=s\left(\sum_{i} p^{i} \Phi^{-1}\left(v_{(1)}^{i} S\left(v_{(2)}^{i}\right)\right) \otimes v_{(0)}^{i}\right)\right) \\
& =s\left(\sum_{i} p^{i} \otimes v^{i}\right) \text {, } \\
& \left(s \circ \widetilde{R}_{b \otimes 1}=R_{b} \circ s\right) \\
& \text { (see Lemma A.7) } \\
& \left(\Phi^{-1}(1)=1\right)
\end{aligned}
$$

where

$$
\mathrm{F}:=\left(m_{P} \otimes i d\right) \circ\left(i d \otimes\left(\Phi^{-1} \circ m\right) \otimes i d\right) \circ(i d \otimes i d \otimes \tau) \circ\left(i d \otimes\left(\tau \circ \rho_{R}\right) \otimes i d\right) .
$$

Furthermore, it is straightforward to check that $\Psi \circ \widetilde{\Psi}=i d$. Hence, $\widetilde{\Psi}$ is the inverse of $\Psi$, as needed.

\section{B Axiomatic Definition of a Frame Bundle}

The theorem below allows an axiomatic definition of a frame bundle. This theorem should have been proven many years ago. Nevertheless, since no appropriate reference has been found, we include a proof.

Theorem B.1 Let $M$ be a smooth manifold with $\operatorname{dim} M=n \in \mathbb{N}$. A principal fiber bundle $P(M, G L(n, \mathbb{R}))$ is isomorphic to the frame bundle $F M$ if and only if there exists a smooth $\mathbb{R}^{n}$-valued id-equivariant 1 -form $\theta$ on $P$ such that $\operatorname{Ker} \theta=\operatorname{Ker} \pi_{*}$, where $\pi: P \rightarrow M$ is the bundle projection.

Remark B.2 Here, by an isomorphism of two principal fiber bundles $P(M, G)$ and $P^{\prime}(M, G)$ we understand a diffeomorphism $f: P \rightarrow P^{\prime}$ satisfying $\pi^{\prime} \circ f=\pi$ and $f \circ R_{g}=R_{g}^{\prime} \circ f$ for any $g \in G$. 
Proof of Theorem B.1. It is clear that if $P(M, G L(n, \mathbb{R}))$ is isomorphic to $F M$, then the canonical 1-form on $F M$ yields, via the bundle isomorphism, the desired 1-form on $P$. Conversely, assume that $P$ admits $\theta$ satisfying the above conditions. Then, remembering that a coframe at any point $m \in M$ is a linear isomorphism from $T_{m} M$ to $\mathbb{R}^{n}$, we can construct the following map:

$$
\begin{aligned}
& J: P \stackrel{\breve{J}}{\longrightarrow} F^{*} M \longrightarrow F M \\
& J: p \longmapsto \sigma^{*} \theta_{p} \longmapsto\left(\sigma^{*} \theta_{p}\right)^{-1},
\end{aligned}
$$

where $\sigma$ is any smooth section of $P$ verifying $(\sigma \circ \pi)(p)=p$. Note that, due to the assumption $\operatorname{Ker} \theta=\operatorname{Ker} \pi_{*}$, the map $\breve{J}$ indeed takes values in the coframe bundle $F^{*} M$ and does not depend on the choice of $\sigma$. Thus, $J$ is uniquely and well-defined. Let $\widetilde{R}: F M \times G L(n, \mathbb{R}) \ni(e, g) \mapsto e \circ g \in F M$ be the right action and $\widetilde{\pi}: F M \rightarrow M$ be the bundle projection. Evidently, $\widetilde{\pi} \circ J=\pi$ and, for any $g \in G L(n, \mathbb{R}), \widetilde{R}_{g} \circ J=J \circ R_{g}$, whence $J$ is a bijection. Now, let $p$ be any point in $P$. There always exists an open neighborhood $U$ of the point $\pi(p)$ over which we can pick smooth sections $\chi: U \rightarrow P$ and $\tilde{\chi}: U \rightarrow F M$. Since $p$ is arbitrary, to prove that $J$ is smooth, it suffices to show that the map $T_{\tilde{\chi}} \circ J \circ T_{\chi}^{-1}: U \times G L(n, \mathbb{R}) \rightarrow U \times G L(n, \mathbb{R})$, where $T_{\tilde{\chi}}$ and $T_{\chi}$ are local trivializations associated with $\tilde{\chi}$ and $\chi$ respectively, is smooth. We have

$$
\left(T_{\tilde{\chi}} \circ J \circ T_{\chi}^{-1}\right)(u, g)=\left(u,\left(\sigma^{*} \theta_{\chi(u)} \circ \widetilde{\chi}(u)\right)^{-1} \circ g\right) .
$$

Since $\sigma^{*} \theta_{p}$ does not depend on the choice of $\sigma$, for any $p \in \chi(U)$, we can pick $\sigma=\chi$. Taking (38) into account, one can see that the smoothness of $J$ follows from the smoothness of the map

$$
\ell: U \ni u \longmapsto \theta_{\chi(u)} \circ \chi_{*} \circ \tilde{\chi}(u) \in G L(n, \mathbb{R}) .
$$

Furthermore, $J^{-1}$ is also smooth because $\left(T_{\chi} \circ J^{-1} \circ T_{\tilde{\chi}}^{-1}\right)(u, g)=\left(u, \sigma^{*} \theta_{\chi(u)} \circ \widetilde{\chi}(u) \circ g\right)$. Hence $J$ is a bundle isomorphism and $P(M, G L(n, \mathbb{R}))$ and $F M$ are isomorphic, as claimed.

An advantage of such an axiomatic definition of a frame bundle is that it is, at least a priori, translatable into the language of quantum principal bundles. More precisely, we could try to define a quantum frame bundle as a quantum bundle $P(B, A)$ equipped with a horizontal (or strongly horizontal) form $\theta: V \hookrightarrow \Omega^{1}(P)$ satisfying $\Delta_{R} \circ \theta=(\theta \otimes i d) \circ \rho_{R}$, where $\left(V, \rho_{R}\right)$ is an appropriate right $A^{o p}$ (or $A$ ) comodule algebra. (An example of such a construction for the case of a classical group has been provided in [23]; in particular, see Section 4 of [23].) It seems interesting to consider in this context bundles over the quantum sphere $S_{q}^{2}$ (see [29] or [9]) with $A=G L_{q}(2, \mathbb{R})$ (see p.161 in [36]), $V$ a quantum plane (see [27]) and $\rho_{R}$ the standard right coaction (see Appendix B in [9]).

Acknowledgments. This work was in part supported by the NSF grant 1-443964-21858-2. Writing up the revised version was partially supported by the KBN grant 2 P301 02007 and by a visiting fellowship at the International Centre for Theoretical Physics in Trieste. I am particularly indebted to Tomasz Brzeziński and Marc Rieffel, whose manifold and generous assistance facilitated the preparation of this article. I also would like to express my gratitude to Shahn Majid, Markus Pflaum, Nicolai Reshetikhin, Philip Ryan, Shuzhou Wang and Stanisław Zakrzewski for helpful discussions, and to Shoshichi Kobayashi and Joseph Wolf for consultations on Theorem B.1. 


\section{References}

[1] Abe, E.: Hopf Algebras. Cambridge University Press 1980

[2] Aref'eva, I.Ya., Arutyunov, G.E.: Uniqueness of $U_{q}(N)$ as a quantum gauge group and representations of its differential algebra. (hep-th/9305176)

[3] Aref'eva, I.Ya., Volovich, I.V.: Quantum group gauge fields. Mod. Phys. Lett. A 6 (10), 893-907 (1991)

[4] Booss, B., Bleecker, D.D.: Topology and Analysis. The Atiyah-Singer Formula and Gauge-Theoretic Physics. Springer-Verlag 1985

[5] Bourbaki, N.: Algèbre Homologique. Masson 1980

[6] Brzeziński, T.: Differential Geometry of Quantum Groups and Quantum Fibre Bundles. Ph.D. thesis, Cambridge University 1994

[7] Brzeziński, T.: Translation Map in Quantum Principal Bundles. to appear in J. Geom. Phys. (hep-th/9407145)

[8] Brzeziński, T., Majid, S.: Quantum Group Gauge Theory on Classical Spaces. Phys. Lett. B 298, 339-43 (1993) (hep-th/9210024)

[9] Brzeziński, T., Majid, S.: "Quantum Group Gauge Theory on Quantum Spaces" Commun. Math. Phys. 157, 591-638 (1993) (hep-th/9208007); erratum Commun. Math. Phys. 167, 235 (1995)

[10] Budzyński, R.J., Kondracki, W.: Quantum Principal Fiber Bundles: Topological Aspects. (hep-th/9401019)

[11] Connes, A.: The Action Functional in Non-Commutative Geometry. Commun. Math. Phys. 117, 673-83 (1988)

[12] Connes, A.: Non-Commutative Geometry. Academic Press 1994

[13] Connes, A., Rieffel, M.: Yang-Mills for Non-Commutative Two-Tori. Contemp. Math. 62, 237-66 (1987)

[14] Coquereaux, R.: Noncommutative Geometry and Theoretical Physics. J. Geom. Phys. 6, (3) 425-90 (1989)

[15] Doebner, H.D., Hennig, J.D., Lücke, W. Mathematical Guide to Quantum Groups. In: Doebner, H.D., Hennig, J.D. (eds.) Quantum Groups. Proceedings of the 8th International Workshop on Mathematical Physics, Clausthal, West Germany, 19-26 July 1989. pp. 29-63. Springer-Verlag 1990

[16] Dubois-Violette, M.: Dérivations et calcul différentiel non commutatif. C. R. Acad. Sci. Paris 307 (Série I), 403-8 (1988)

[17] Dubois-Violette, M., Kerner, R., Madore, J.: Noncommutative Differential Geometry of Matrix Algebras. J. Math. Phys. 31 (2), 316-22 (1990)

[18] Dubois-Violette, M., Kerner, R., Madore, J.: Noncommutative Differential Geometry and New Models of Gauge Theory. J. Math. Phys. 31 (2), 323-30 (1990)

[19] Durdevic, M.: Geometry of Quantum Principal Bundles I. to appear in Commun. Math. Phys. (a-alg/9507019)

[20] Durdevic, M.: Geometry of Quantum Principal Bundles II (Extended Version). (a-alg/9412005)

[21] Durdevic, M.: Quantum Principal Bundles and Corresponding Gauge Theories. (q-alg/9507021)

[22] Durdevic, M. Quantum Principal Bundles. In: Proceedings of the XXII International Conference on Differential Geometric Methods in Theoretical Physics. Ixtapa, Mexico 1993 (hep-th/9311029)

[23] Durdevic, M.: On Framed Quantum Principal Bundles. (q-alg/9507020)

[24] Fadeev, L.D.,Reshetikhin, N.Yu., Takhtadzhyan, L.A.: Quantization of Lie Groups and Lie Algebras. Leningrad Math. J. 1 (1), 193-225 (1990)

[25] Hazewinkel, M.: Formal Groups and Applications. Academic Press 1978

[26] Majid, S.: Introduction to Braided Geometry and q-Minkowski space. In: Castellani, L., Wess, J. (eds.) Proceedings of the International School of Physics 'Enrico Fermi' CXXVII. IOS Press 1996 (hep-th/9410241) 
[27] Manin, Yu.I.: Quantum Groups and Noncommutative Geometry. Publications du CRM 1561, Univ. de Montreal 1988

[28] Pflaum, M.J.: Quantum Groups on Fibre Bundles. Commun. Math. Phys. 166 (2), 279-315 (1994) (hep-th/9401085

[29] Podleś, P.: Quantum Spheres. Lett. Math. Phys. 14, 521-31 (1987)

[30] Rieffel, M.: Critical Points of Yang-Mills for Noncommutative Two-Tori. J. Differential Geom. 31, 535-46 (1990)

[31] Schneider, H.J.: Principal Homogenous Spaces for Arbitrary Hopf Algebras. Isr. J. Math. 72 (1-2), 167-95 (1990)

[32] Schneider, H.J.: Hopf Galois Extensions, Crossed Products, and Clifford Theory. In: Bergen, J., Montgomery, S. (eds.) Advances in Hopf Algebras. Lecture Notes in Pure and Applied Mathematics. 158, Marcel Dekker, Inc. 1994

[33] Shnider, S., Sternberg, S.: Quantum Groups. International Press Inc. 1993

[34] Spera, M.: A Symplectic Approach to Yang-Mills Theory for Non Commutative Tori. Can. J. Math. 44 (2), 368-87 (1992)

[35] Sweedler, M.E.: Hopf Algebras. Benjamin 1969

[36] Takhtadzhyan, L.A. Lectures on Quantum Groups. In: Introduction to Quantum Groups and Integrable Massive Models of Quantum Field Theory. pp. 69-197. World Scientific 1990

[37] Trautman, A.: Differential Geometry for Physicists. Bibliopolis 1984

[38] Woronowicz, S.L.: Compact Matrix Pseudogroups. Commun. Math. Phys. 111, 613-65 (1987)

[39] Woronowicz, S.L.: Differential Calculus on Compact Quantum Pseudogroups (Quantum Groups). Commun. Math. Phys. 122, 125-70 (1989)

[40] Wu, K., Zhang, R.-J.: Algebraic approach to gauge theory and its noncommutative extension. Commun. Theor. Phys. 17 (2), 175-82 (1992) 